\title{
THE STABLE GEOMETRIC DIMENSION OF VECTOR BUNDLES OVER REAL PROJECTIVE SPACES
}

BY

DONALD M. DAVIS, SAM GITLER AND MARK MAHOWALD ${ }^{1}$

\begin{abstract}
An elementary argument shows that the geometric dimension of any vector bundle of order $2^{e}$ over $R P^{n}$ depends only on $e$ and the residue of $n \bmod 8$ for $n$ sufficiently large. In this paper we calculate this geometric dimension, which is approximately $2 e$. The nonlifting results are easily obtained using the spectrum $b J$. The lifting results require bo-resolutions. Half of the paper is devoted to proving Mahowald's theorem that beginning with the second stage bo-resolutions act almost like $K\left(Z_{2}\right)$-resolutions.
\end{abstract}

1. Introduction. Let $P_{k}^{l}$ denote the stunted real projective $R P^{l} / R P^{k-1}$. In the stable range $(l+8<2 k-1)$ there is a map $P_{k+8}^{l+8} \rightarrow^{\varphi} P_{k}^{l}$ (when $k$ is odd and $l$ even) which induces an isomorphism in $K O$-theory. Thus if $f: P_{k}^{l} \rightarrow B O$ classifies a (stable) vector bundle of order $2^{e}$ in $(K O)^{\sim}\left(P^{l}\right)$, then $f \varphi$ classifies a bundle of order $2^{e}$ in $(K O)^{\sim}\left(P^{l+8}\right)$. Since the geometric dimension $\operatorname{gd}(\theta)$ of a stable vector bundle $\theta$ is the smallest $r$ such that its classifying map lifts to $B O_{r}$, this implies that for fixed $e$ the gd of a bundle of order $2^{e}$ over $P^{n}$ is a nonincreasing function of $n$ for $n$ in a fixed mod 8 congruence class, and hence must achieve a stable value. In this paper we compute this stable value for almost every $e$.

THEOREM 1.1. (i) If $e \geqslant 20$ and $n$ is sufficiently large, the geometric dimension of any vector bundle of order $2^{e}$ over $P^{n}$ is $2 e+\delta(n, e)$, where $\delta$ is given by

\begin{tabular}{ccccccccccc}
\multicolumn{1}{c}{$e \bmod 4$} & \multicolumn{1}{c}{$n \bmod 8$} \\
\cline { 3 - 12 } & 0 & 0 & 1 & 2 & 3 & 4 & 5 & 6 & 7 \\
\hline & 1 & 2 & 0 & 0 & 0 & 0 & 0 & 0 & 0 \\
& 2 & 2 & 0 & -1 & -1 & -1 & 0 & 2 & 2 \\
& 3 & 1 & 0 & -2 & -2 & -2 & 0 & 1 & 1
\end{tabular}

(ii) For any $e$ and $n$ the geometric dimension of any vector bundle of order $2^{e}$ over $P^{n}$ is $\geqslant 2 e+\delta(n, e)$.

1.1(i) can be proved by these methods for some smaller values of $e$ (see Remark 4.3), but for some very small values of $e$, the stable geometric dimension is greater

Received by the editors March 6, 1980.

1980 Mathematics Subject Classification. Primary 55G35, 55G40; Secondary 55B15, 55B20.

Key words and phrases. Geometric dimension of vector bundles, real projective space, obstruction theory, bo-resolutions.

${ }^{1}$ First and third authors were supported by N.S.F. grants. 
than $2 e+\delta(n, e)$. The condition " $n$ sufficiently large" can be made precise as $n \geqslant 4 e+30+4\left[\log _{2}(e+4)\right]$.

The nonlifting results ( $\geqslant$ part) are virtually identical to those of every other approach to the geometric dimension question which utilizes, however indirectly, the Adams operation $\psi^{3}-1$ ([4], [9], [6], [7]). In certain congruences our results are slightly stronger because the results were overlooked in [4], and [9] did not consider $n$ odd.

The significance of our work is that these $b J$-primary obstructions are a total obstruction for the lifting questions dealt with in 1.1(i). It shows that our control over homotopy theory as applied to this classical geometrical question is rather complete.

REMARK 1.2. Another interpretation of 1.1(i) is that for these bundles the geometric dimension equals the fibre-homotopy geometric dimension (see Remark 2.6).

The most significant and most novel part of the theorem is the lifting results. This represents the first successful attempt to use obstruction theory with arbitrarily many nontrivial obstruction groups. Since the obstruction groups, i.e. homotopy groups of Stiefel manifolds, are known completely only through at most 29 nontrivial dimensions [13], a lifting procedure which works for purely dimensional reasons is clearly required here. This is provided by Mahowald's theory of bo-resolutions [14]. In $\$ 3$ we expand considerably upon the proof given in [14], adapting the result to spaces other than $S^{\circ}$.

Another novel aspect of our lifting results is that we are usually lifting far beyond the metastable range. For example, the case $e=20, n=8 l+4$, says $\operatorname{gd}\left(a \cdot 2^{4 l-17} \xi_{8 l+4}\right)=40$, when $a$ is odd and $l \geqslant 16$. Thus a theory of unstable resolutions is required; this is provided by the $\lambda$-algebera methods of Curtis et al.

2. Nonlifting results. Let $\nu(m)$ denote the exponent of the largest 2-power dividing $m$. In this section we shall prove

THEOREM 2.1. If $n \leqslant 4 \nu(m)-3$, then $\operatorname{gd}\left(m \xi_{n}\right) \geqslant n-2 \nu(m)+\varepsilon(n, \nu(m))$ where $\varepsilon$ is given by the table

\begin{tabular}{cc|cccc}
\multicolumn{1}{c}{} & \multicolumn{5}{c}{$n \bmod 4$} \\
\multirow{4}{*}{$\nu(m) \bmod 4$} & 0 & 1 & 2 & 3 \\
\cline { 3 - 6 } & 1 & 0 & 1 & 1 & 0 \\
& 2 & 1 & 1 & 2 & 1 \\
& 3 & 2 & 1 & 2 & 1 \\
& & & 1 & 0 & -1
\end{tabular}

Since the order of $m \xi_{n}$ in $(K O)^{\sim}\left(P^{n}\right)$ is $2^{\varphi(n)-\nu(m)}$, where $\varphi(n)$ is the number of positive integers $\leqslant n$ which are $\equiv 0,1,2$, or $4(8)$, Theorem 2.1 readily implies that the numbers in 1.1 are lower bounds.

Let $V_{r}=S O / S O_{r}$ and $P_{r}=R P^{\infty} / R P^{r-1}$. Let $Q X=\Omega^{\infty} \Sigma^{\infty} X$. The functor $Q$ may be considered as the composite of the functor $\Sigma^{\infty}$ : Spaces $\rightarrow$ Spectra which forms the suspension spectrum with the functor $\Omega^{\infty}:$ Spectra $\rightarrow$ Spaces which takes 
the 0 th space in the associated $\Omega$-spectrum. The inclusion $P_{r} \rightarrow V_{r}$ admits a stable retraction $V_{r} \rightarrow Q P_{r}$. This follows from early work of James. See, for example, [19, \4.2].

Let $b o$ denote the spectrum for connective $K O$-theory localized at $2, \Sigma^{4} b s p$ the spectrum obtained from $b o$ by killing $\pi_{i}$ for $i<4$, and $b J$ the fibre of the Adams operation $\theta=\psi^{3}-1:$ bo $\rightarrow \Sigma^{4} b s p$ [16], [5]. The map $\Sigma^{\infty} P_{r} \rightarrow P_{r} \wedge b J$ induces a $\operatorname{map} Q P_{r} \rightarrow \Omega^{\infty}\left(P_{r} \wedge b J\right)$.

If $n \leqslant 4 \nu(m)-3$ (the hypothesis of 2.1 ), $m \xi_{n}$ is trivial on a skeleton of dimension $b-1>n / 2$, so that its classifying map $f_{m}$ factors through $P_{b}^{n}$, which has a desuspension $\Sigma^{-1} P_{b}^{n}$. Adjoint to $f$ is a map $\Sigma^{-1} P_{b}^{n} \rightarrow^{m \xi} S O$. 2.1 will be proved by showing that if $r=n-2 \nu(m)+\varepsilon-1$, the composite

$$
\Sigma^{-1} P_{b}^{n} \stackrel{m \xi}{\rightarrow} S O \rightarrow S O / S O_{r}=V_{r} \rightarrow Q P_{r} \rightarrow \Omega^{\infty}\left(P_{r} \wedge b J\right)
$$

is essential.

If $X$ is a space and $b$ is a spectrum, $[X, b]$ will denote $\left[\Sigma^{\infty} X, b\right] \approx\left[X, \Omega^{\infty} b\right]$. In the exact sequence below, the isomorphism $\left[\Sigma^{-1} X, \Sigma^{-1} b\right] \approx[X, b]$ has been used in the first two groups.

$$
\begin{aligned}
{\left[P_{b}^{n}, P_{r} \wedge b o\right] } & \stackrel{\theta_{*}}{\rightarrow}\left[P_{b}^{n}, P_{r} \wedge \Sigma^{4} b s p\right] \\
& \rightarrow\left[\Sigma^{-1} P_{b}^{n}, P_{r} \wedge b J\right] \rightarrow\left[\Sigma^{-1} P_{b}^{n}, P_{r} \wedge b o\right] .
\end{aligned}
$$

The groups $\pi_{*}\left(P_{r} \wedge \Sigma^{4} b s p\right)$ have been calculated, e.g. in [6, 3.4], to be given by charts which begin as below and continue similarly. In these charts dots indicate nonzero elements, vertical lines multiplication by 2 , diagonal lines multiplication by $\eta \in \pi_{1}\left(S^{\circ}\right)$, and an arrow an infinite tower. Thus for example

$$
\pi_{4 k+11}\left(P_{4 k-1} \wedge \Sigma^{4} b s p\right) \approx \mathbf{Z}_{32} \text { and } \pi_{4 k+4}\left(P_{4 k} \wedge \Sigma^{4} b s p\right) \approx \mathbf{Z}_{(2)}
$$

the integers localized at 2 .
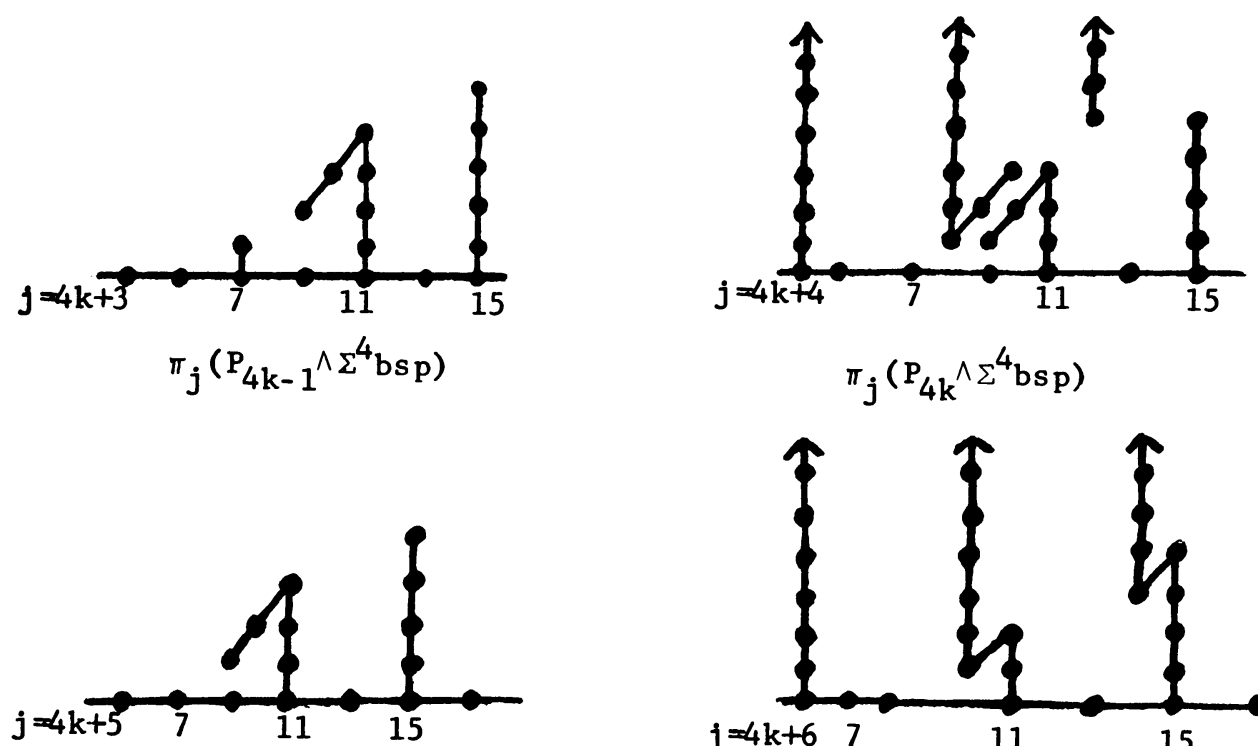

$$
\pi_{j}\left(\mathrm{P}_{4 \mathrm{k}+1^{\wedge}} \wedge \Sigma^{4} \mathrm{bsp}\right)
$$

$$
\pi_{j}\left(\mathrm{P}_{4 \mathrm{k}} \wedge \Sigma^{4} \mathrm{bsp}\right)
$$

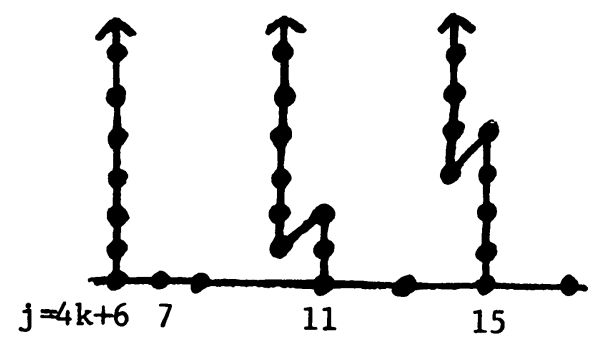

$$
\pi_{j}\left(P_{4 k+2} \wedge \Sigma^{4} b s p\right)
$$


We describe $\left[P_{b}^{n}, P_{r} \wedge \Sigma^{4} b s p\right]$ below in terms of the above charts, in the case $b$ odd, which can always be arranged. It is a sum of $\mathbf{Z}_{2}$ 's of filtration 0 plus the cyclic groups described below. If $n \equiv 3$ (4), $\left[P_{b}^{n}, P_{r} \wedge \Sigma^{4} b s p\right] \approx\left[P_{b}^{n-1}, P_{r} \wedge \Sigma^{4} b s p\right]+$ $\pi_{n}\left(P_{r} \wedge \Sigma^{4} b s p\right)$, so for the rest of the paragraph we shall assume $n \neq 3$ (4). If $r$ is odd there is one cyclic summand, with a class for each element of maximal filtration in $\pi_{j}\left(P_{r} \wedge \Sigma^{4} b s p\right)$ with $j \equiv 1,2,3,7(8)$ and $b<j \leqslant n$. Note that this puts one element in each filtration through a range of filtrations. If $r \equiv 2(4)$, there is one cyclic summand, with a class for an element of maximal filtration in $\pi_{j}\left(P_{r} \wedge \Sigma^{4} b s p\right)$ for each $j \equiv 3$ (4) with $b \leqslant j<n$ and a class for an element of minimal filtration in $\pi_{j}\left(P_{r} \wedge \Sigma^{4} b s p\right)$ with $j \equiv 2$ (4) and $b<j \leqslant n$. If $r \equiv O(4)$, $P_{r} \wedge \Sigma^{4} b s p \simeq S^{r} \wedge \Sigma^{4} b s p \vee P_{r+1} \wedge \Sigma^{4} b s p$. Each part of this splitting contributes a cyclic summand, the latter of which was described above. There is a summand with a class for an element of minimal filtration in each $\pi_{j}\left(S^{r} \wedge \Sigma^{4} b s p\right)$ with $j \equiv$ $0,1,2,4(8)$ and $b \leqslant j \leqslant n$.

For example, $\left[P_{13}^{20}, P_{5} \wedge \Sigma^{4} b s p\right] \approx \mathbf{Z}_{2}+\mathbf{Z}_{2}+\mathbf{Z}_{16}$ with the filtration zero $\mathbf{Z}_{2}$ 's corresponding to the elements in boxes in the chart below, and the $\mathbf{Z}_{16}$, generated by a filtration 1 class, corresponding to the classes circled in the chart below.

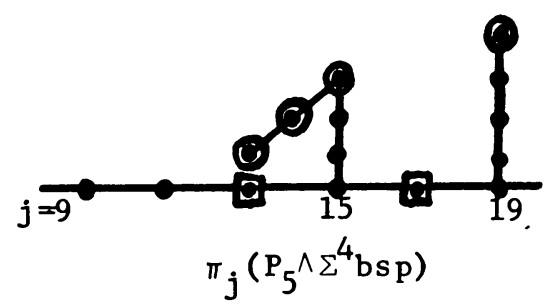

By similar methods one shows that $\left[\Sigma^{-1} P_{b}^{n}, P_{r} \wedge b o\right]$ is a sum of filtration zero $\mathbf{Z}_{2}$ 's. If $n \neq 3$ (4) and $r \neq 0$ (4), the homomorphism $\theta_{*}$ in (2.3) is zero by [6, p. 152], and hence above filtration zero $\left[P_{b}^{n}, P_{r} \wedge \Sigma^{4} b s p\right] \rightarrow\left[\Sigma^{-1} P_{b}^{n}, P_{r} \wedge b J\right]$ is an isomorphism which increases filtration by 1 . The element of order 2 in the additional summand of $\left[P_{b}^{n}, P_{r} \wedge \Sigma^{4} b s p\right]$ when $n \equiv 3$ (4) or $r \equiv 0$ (4) is hit by $\theta_{*}$ unless $\nu(n+1) \geqslant \varphi(n-r)$ or $\nu(r) \geqslant \varphi(n-r)$.

Proposition 2.4. The composite (2.2) is essential if and only if there is a nonzero class of filtration $\nu(m)-3$ in

$$
\begin{cases}{\left[P_{b}^{n-1}, P_{r} \wedge \Sigma^{4} b s p\right]} & \text { if } n \equiv 3(4) \text { and } \nu(n+1)<\varphi(n-r) \\ {\left[P_{b}^{n}, P_{r+1} \wedge \Sigma^{4} b s p\right]} & \text { if } r \equiv 0(4) \text { and } \nu(r)<\varphi(n-r) \\ {\left[P_{b}^{n}, P_{r} \wedge \Sigma^{4} b s p\right]} & \text { otherwise. }\end{cases}
$$

ProOF.

$$
\begin{aligned}
(K O)^{\sim}\left(P_{b}^{n}\right) & \approx\left[\Sigma^{-1} P_{b}^{n}, S O\right] \rightarrow\left[\Sigma^{-1} P_{b}^{n}, Q(S O)\right] \\
& \rightarrow\left[\Sigma^{-1} P_{b}^{n}, Q\left(P_{1}\right)\right] \rightarrow\left[\Sigma^{-1} P_{b}^{n}, P_{1} \wedge b J\right] \\
& \approx\left[P_{b}^{n}, P_{1} \wedge \Sigma^{4} b s p\right] \rightarrow\left[P_{b}^{n}, P_{r} \wedge \Sigma^{4} b s p\right]
\end{aligned}
$$


are all homomorphisms, since the homotopy and Whitney sums must agree on [ , $Q(S O)$ ]. Thus divisibility by 2 in $(K O)^{\sim}\left(P^{n}\right)$ corresponds to filtration in $\left[P^{n}, P_{r} \wedge \Sigma^{4} b s p\right]$.

If $a$ is any odd integer, $16 a \xi_{8}$ is trivial and $16 a \xi_{9}$ is nontrivial but not detected by Stieffel-Whitney classes. Thus the element of $\left[P^{n}, P_{1} \wedge \Sigma^{4} b s p\right]$ corresponding to $16 a \xi$ is trivial on $P^{8}$ and detected on the 9-cell by the filtration 1 class in $\pi_{9}\left(P_{1} \wedge \Sigma^{4} b s p\right)$. Thus by additivity $2^{e} a \xi$ corresponds to a class of filtration $e-3$.

When $n \equiv 3$ (4), the class of $2^{e} a \xi$ in $\left[P^{n}, P_{r} \wedge \Sigma^{4} b s p\right]$ is the sum of the filtration $e-3$ classes in both summands. This follows since the map extends over $P^{n+1}$, and $\left[P^{n+1}, P_{r} \wedge \Sigma^{4} b s p\right] \rightarrow\left[P^{n}, P_{r} \wedge \Sigma^{4} b s p\right]$ maps to the sums. This is important because the $\pi_{n}\left(P_{r} \wedge \Sigma^{4} b s p\right)$-summand extends one filtration higher than $\left[P^{n-1}, P_{r}\right.$ $\wedge \Sigma^{4} b s p$ ], giving stronger results if the top class is not in the image of $\boldsymbol{\theta}_{*}$.

Similarly if $r \equiv 0$ (4), [ $\left.P^{n}, P_{r} \wedge \Sigma^{4} b s p\right]$ has a summand due to the $r$-cell which extends one filtration higher than its $\left[P^{n}, P_{r+1} \wedge \Sigma^{4} b s p\right]$-summand. This is utilized effectively if it is not in the image of $\theta_{*}$ because $\left[P^{n}, P_{1} \wedge \Sigma^{4} b s p\right] \rightarrow\left[P^{n}, P_{r} \wedge\right.$ $\left.\Sigma^{4} b s p\right]$ maps onto the sum of the two summands when $r \equiv 0$ (4).

From the charts of $\pi_{*}\left(P_{r} \wedge \Sigma^{4} b s p\right)$ and the discussion of how to determine $\left[P^{n}, P_{r} \wedge \Sigma^{4} b s p\right]$ from them, we tabulate

Proposition 2.5. If $n \neq 3$ (4), the largest $r \neq 0$ (4) such that $\left[P^{n}, P_{r} \wedge \Sigma^{4} b s p\right]$ contains a nonzero class in filtration $e-3$ is $n-2 e+\varepsilon(n, e)-1$, where $\varepsilon$ is as in 2.1 .

2.1 follows immediately from 2.4 and 2.5 .

REMARK 2.6. Let $S G_{r}$ denote the space of degree 1 maps $S^{r-1} \rightarrow S^{r-1}$ and $S G=\cup_{r} S G_{r} . B S G_{r}$ is the classifying space for fiber homotopy equivalence classes of $S^{r-1}$-fibrations. There is a map of fibrations

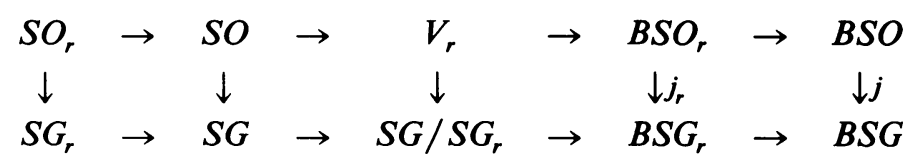

in which $j$ corresponds to taking the sphere bundle of a vector bundle, and the fiber-homotopy geometric dimension of a stable spherical fibration is the smallest $r$ such that the classifying map lifts to $B S G_{r}$. Since the map $V_{r} \rightarrow Q P_{r}$ utilized in (2.2) factors through $S G / S G_{r}$, Remark 1.2 follows immediately from the fact that in the cases covered by 1.1(i) a map $\Sigma^{-1} P^{n} \rightarrow V_{r}$ is null-homotopic if and only if its composite into $\Omega^{\infty}\left(P_{r} \wedge b J\right)$ is null-homotopic.

3. Resolutions. In this section we will prove some results about (unstable) Adams resolutions and stable bo-resolutions which will be used in $\$ 4$ to prove the lifting part of Theorem 1.1.

Definition 3.1. Let $X$ be a space of finite type. A resolution of $X$ is a diagram

\begin{tabular}{|c|c|c|c|c|c|}
\hline$X$ & $\stackrel{p_{0}}{\leftarrow}$ & $X^{\langle 1\rangle}$ & $\stackrel{p_{1}}{\leftarrow}$ & $X^{\langle 2\rangle}$ & $\leftarrow$ \\
\hline$\downarrow i_{0}$ & & $\downarrow i_{1}$ & & $\downarrow i_{2}$ & \\
\hline$K_{0}$ & & $K_{1}$ & & $K_{2}$ & \\
\hline
\end{tabular}


where $K_{i}$ is a product of Eilenberg-Mac Lane spaces $K\left(\mathbf{Z}_{2}, n\right)$ for $i>0$, and $K_{0}$ is a product of $K\left(\mathbf{Z}_{2}, n\right)$ 's and possibly also $K(\mathbf{Z}, n)$ 's, and $\Omega K_{s-1} \rightarrow X^{\langle s\rangle} \rightarrow^{p_{s}} X^{\langle s-1\rangle}$ is the fibration classified by $i_{s-1}$. If also $i_{s}^{*}: H^{*}\left(K_{s}\right) \rightarrow H^{*}\left(X^{\langle s\rangle}\right)$ is surjective for all $s$, this is an Adams resolution. (Here and throughout the paper all cohomology groups have $\mathbf{Z}_{2}$-coefficients unless indicated otherwise.) An Adams resolution through dimension $d$ is a resolution in which the $K\left(\mathbf{Z}_{2}, n\right)$ 's have $n \leqslant d$ and $i_{s}^{*}$ is surjective through dimension $d$. An Adams resolution of a spectrum is defined similarly. If $X$ is a space, then applying $\Omega^{\infty}()$ to an Adams resolution of the spectrum $\Sigma^{\infty} X$ yields a resolution

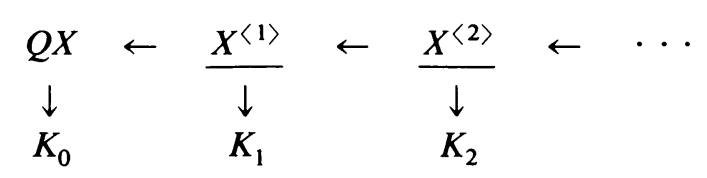

which may not be an Adams resolution but satisfies $E_{2}^{s, t} \approx \operatorname{Ext}_{\mathscr{Q}}^{s, t}\left(H^{*} X, \mathbf{Z}_{2}\right)$.

If $\pi_{*}()$ is applied to a resolution of $X$, a spectral sequence is obtained with $E_{1}^{s, t+s}=\pi_{t}\left(K_{s}\right)$ and $E_{\infty}^{*, t+*}$ an associated graded group to $\pi_{t}(X)$ if $X^{\langle\infty\rangle}$ is acyclic.

Proposition 3.2. (i) If $f: X \rightarrow Y$ is a map, $\left\{X^{\langle s\rangle}\right\}$ is an Adams resolution, and $\left\{Y^{\langle s\rangle}\right\}$ is a resolution, there exists a map of resolutions $\left\{X^{\langle s\rangle} \rightarrow Y^{\langle s\rangle}\right\}$.

(ii) If $\left\{X^{\langle s\rangle} \rightarrow Y^{\langle s\rangle}\right\}$ is a map of resolutions covering a map $f: X \rightarrow Y$, then there is a resolution of the fiber $F$ of $f$ such that there is an exact sequence

$$
\cdots \rightarrow E_{2}^{s, t}(F) \rightarrow E_{2}^{s, t}(X) \rightarrow E_{2}^{s, t}(Y) \rightarrow E_{2}^{s+1, t}(F) \rightarrow \cdots
$$

Proof of (ii). Let $F^{\langle s\rangle}$ be the fiber of $X^{\langle s\rangle} \rightarrow Y^{\langle s-1\rangle}$. Then $F^{\langle s+1\rangle} \rightarrow F^{\langle s\rangle}$ is classified by $K_{s}(X) \times \Omega K_{s-1}(Y)$, so that $0 \rightarrow E_{1}^{s-1, t}(Y) \rightarrow E_{1}^{s, t}(F) \rightarrow E_{1}^{s, t}(X) \rightarrow 0$ is exact.

THEOREM 3.3. Suppose $\left\{g_{s}\right\}$ is the map of resolutions induced by the map $V_{r} \rightarrow Q P_{r}$ considered in $\$ 2$. In the diagram

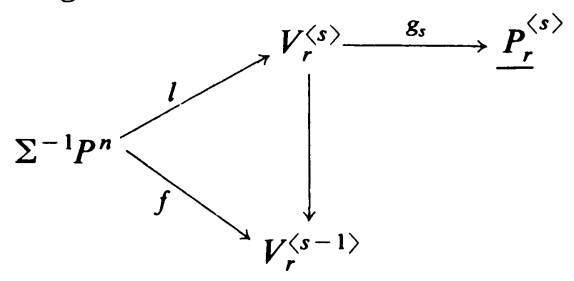

if $n \leqslant 2 r+2 s-4$ and $\left[g_{s} \circ l\right]=0$, then $[f]=0$.

Proof. Let $X_{r+i, i}$ denote the fibre of $V_{r+i, i} \rightarrow Q\left(P_{r}^{r+i-1}\right)$,

$$
X_{r}=\operatorname{fibre}\left(V_{r} \rightarrow Q\left(P_{r}\right)\right)
$$

and $F_{r+i}=\operatorname{fibre}\left(S^{r+i} \rightarrow Q\left(S^{r+i}\right)\right)$. There is a diagram

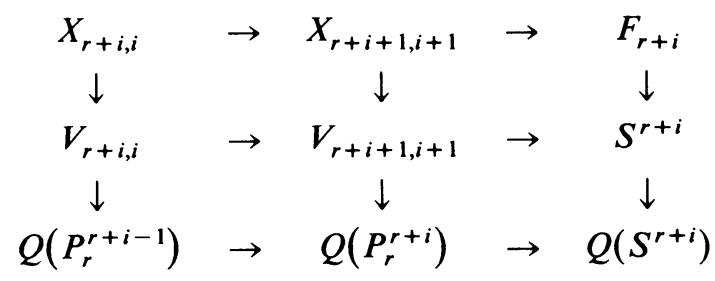


in which the rows and columns are fibrations. By 3.2(ii) resolutions can be chosen so that these fibrations induce long exact sequences in $E_{2}^{*, *}$. By [3], $E_{2}^{s, s+j}\left(F_{m}\right)=0$ if $j<2 m+2 s-2$, except for $E_{2}^{s, s+2 m-1}\left(F_{m}\right)=\mathbf{Z}_{2}$ if $m$ is even. Since $X_{r}$ $=\lim _{\rightarrow i} X_{r+i, i}$ and $X_{r+1,1}=F_{r}$, the exact sequences in $E_{2}^{* * *}$ obtained from the first row of the above diagram for all $i \geqslant 1$ show that $E_{2}\left(X_{r}\right)$ is built up from $E_{2}\left(F_{m}\right)$ for $m>r$. Hence by Curtis's vanishing theorem above, if $j<2 r+2 s-2$,

$$
E_{2}^{s, s+j}\left(X_{r}\right)= \begin{cases}\mathbf{Z}_{2}, & j \equiv 3(4), j \geqslant 2 r-1, \\ 0, & \text { otherwise. }\end{cases}
$$

Pictorially, the $E_{2}$-chart for $X_{r}$ with the usual $(t-s, s)$ grading is

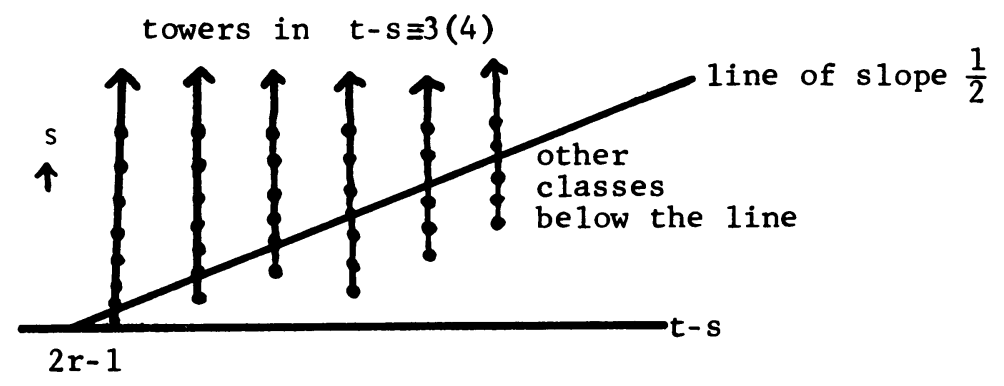

The exact sequence in $E_{2}$ induced by $X_{r} \rightarrow V_{r} \rightarrow Q\left(P_{r}\right)$ now implies that $E_{2}\left(V_{r}\right)$ has the following rough form:

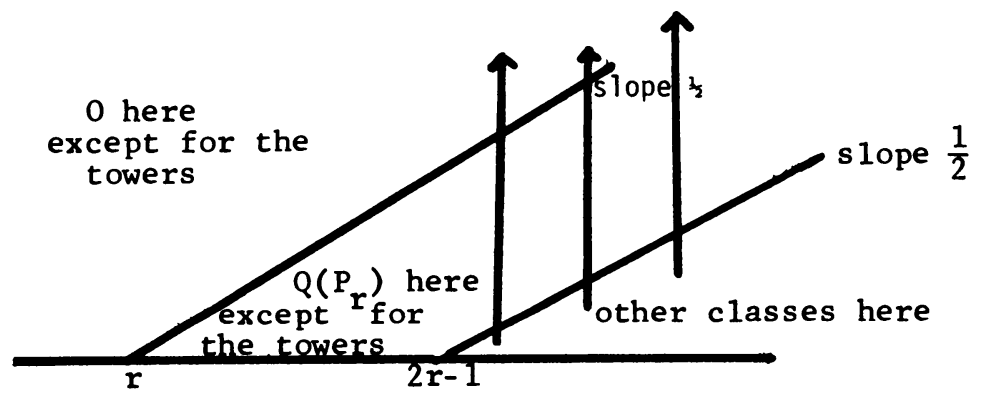

If the composite $\Sigma^{-1} P^{n} \rightarrow^{l} V_{r}^{\langle s\rangle} \rightarrow^{\delta_{c}} P_{r}^{\langle s\rangle}$ is null-homotopic, then $l$ factors through $\Sigma^{-1} P^{n} \rightarrow X_{r}^{\langle s\rangle} \rightarrow^{i} V_{r}^{\langle s\rangle}$. Through dimension $n-1$,

$$
X_{r}^{\langle s\rangle}=\prod_{j>2 r-1 ; j \equiv 3(4)} K(\mathbf{Z}, j),
$$

and the map $X_{r}^{\langle s\rangle} \rightarrow X_{r}^{\langle s-1\rangle}$ is multiplication by 2 on each $K(\mathbf{Z}, j)$. Thus the composite $\Sigma^{-1} P^{n} \rightarrow X_{r}^{\langle s\rangle} \rightarrow X_{r}^{\langle s-1\rangle}$ is the image of

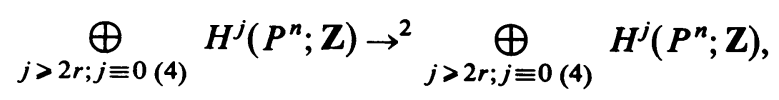

which is 0 .

REMARK 3.3.1. Theorem 3.3 can be strengthened slightly by using the fact that $F_{m}$ is $\Sigma^{m-1} P_{m}$ through a range, so that in 3.3: if $\operatorname{Ext}_{\mathscr{Q}}^{s, t}\left(H^{*}\left(\Sigma^{r-1} P_{r}\right), \mathbf{Z}_{2}\right)=0$ for $2 r-1<t-s \leqslant n-1$ and $\left[g_{s} \circ l\right]=0$, then $[f]=0$. This upper edge of Ext is known from [1] or [13]. 
TheOREM 3.4. If $f: \Sigma^{-1} P_{(n+1) / 2}^{n} \rightarrow S O$ classifies $m \xi$, then $f$ lifts to $S O^{\left\langle\nu(m)-\left[\log _{2} n\right]\right\rangle}$.

Proof. If $0 \leqslant b \leqslant 3$, let $\rho(4 a+b)=8 a+2^{b}$; thus $\rho(n)$ is the $(n+1)$ st positive integer $\equiv 0,1,2$, or $4(8)$. If $X$ is any space or spectrum, let $X[j] \rightarrow X$ denote a map from a $(j-1)$-connected space which induces an isomorphism in $\pi_{i}()$ for $i \geqslant j$. We shall need

LEMMA $3.5[11] . H^{i}(S O[\rho(k)-1]) \rightarrow H^{i}(S O[\rho(k+1)-1])$ is 0 for $i<2^{k+1}-$ 1.

We form the unstable Adams resolution of $S O$ through dimension $n-1$. There is a commutative diagram (where $l=\left[\log _{2} n\right]$ )

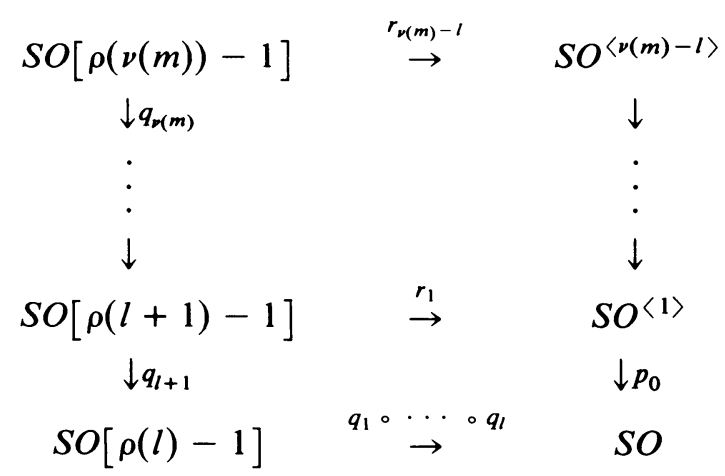

which exists because, by $3.5, H^{i}\left(q_{j}\right)=0$ for $i \leqslant n-1$ and $j>l$, and each $p_{j}$ is induced by a map into Eilenberg-Mac Lane spaces with generators in degree $\leqslant n-1$. Since $m \xi$ is trivial on $P^{\rho(\nu(m))-1}, f$ lifts to $S O[\rho(\nu(m))-1]$. Following by $r_{\nu(m)-l}$ yields the result.

If $X$ is a space, the $b o$-resolution of $X$ is the diagram of spectra

\begin{tabular}{|c|c|c|c|c|c|}
\hline$\Sigma^{\infty} X$ & $\stackrel{q_{0}}{\leftarrow}$ & $X_{1}$ & $\stackrel{q_{1}}{\leftarrow}$ & $X_{2}$ & $\stackrel{q_{2}}{\leftarrow}$ \\
\hline$\downarrow j_{0}$ & & $\downarrow j_{1}$ & & $\downarrow j_{2}$ & \\
\hline$X \wedge b o$ & & $X_{1} \wedge b o$ & & $X_{2} \wedge b o$ & \\
\hline
\end{tabular}

where $q_{s}$ is the fibration induced by $j_{s}$. Let $I$ denote the fiber of the unit map $\mathcal{\delta} \rightarrow b o$, and $I^{\wedge s}=I \wedge \ldots \wedge I$ with $s$ factors. Then $X_{s}=X \wedge I^{\wedge s}$. Splicing the homotopy exact sequences of these fibrations yields a cochain complex $C_{i}(X)$ :

$$
\pi_{i}(X \wedge b o) \stackrel{d_{0}}{\rightarrow} \pi_{i}(X \wedge \overline{b o} \wedge b o) \stackrel{d_{1}}{\rightarrow} \pi_{i}\left(X \wedge \overline{b o} \wedge^{2} \wedge b o\right) \stackrel{d_{2}}{\rightarrow} \cdots
$$

where $\overline{b o}=\Sigma I=\operatorname{cofibre}\left(\mathcal{S} \rightarrow^{i} b o\right)$ and $d_{i}=\left(X \wedge \overline{b o}^{\wedge i}\right) \wedge(b o \rightarrow \overline{b o}) \wedge i$.

The main theorem of $b o$-resolutions, adapted to the space $X$, states

THEOREM 3.6. Suppose $X$ is $(m-1)$-connected and there are no nontrivial differentials in the Adams spectral sequence for $X \wedge$ bo through dimension $i$. Then the cohomology of the cochain complex $C_{i}(X)$ satisfies

$$
H^{s}\left(C_{i}(X)\right)= \begin{cases}0, & i<4 s+m, \\ V_{s, i}, & s \geqslant 2, i \geqslant 4 s+m, \\ Q_{i} \oplus V_{1, i}, & s=1, i>4+m,\end{cases}
$$


where $V_{s, i}$ is a $\mathbf{Z}_{2}$-vector space all elements of which are detected in $\mathbf{Z}_{2}$-cohomology, i.e. they have Adams filtration 0 , and

$$
Q_{i}=\operatorname{coker}\left(\pi_{i}(X \wedge b o) \stackrel{\theta_{*}}{\rightarrow} \pi_{i}\left(X \wedge \Sigma^{4} b s p\right)\right),
$$

where $\theta_{*}$ is as in $\S 2$.

The significance of this result is that bo-resolutions have the $b J$-primary obstructions at levels 0 and 1 , and above this they work almost like a $K\left(\mathbf{Z}_{2}\right)$-resolution, but they have the advantage over $K\left(\mathbf{Z}_{2}\right)$-resolutions of increasing the connectivity faster. To be more precise, we have

COROLlaRY 3.6.1. Suppose there are no nontrivial differentials through dimension $N$ in the ASS for $D_{N} Y \wedge X \wedge$ bo where $D_{N} Y$ denotes a stable $N$-dual of a finite complex $Y$. Let $X_{s}$ denote the sth spectrum in the bo-resolution of $X$. If $s \geqslant 2$ and $l$ : $Y \rightarrow X_{s}$ is cohomologically trivial, then there exists a map $l^{\prime}: Y \rightarrow X_{s}$ such that $q_{s-1} l=q_{s-1} l^{\prime}: Y \rightarrow X_{s-1}$ and $l^{\prime}$ lifts to $X_{s+1}$. (Here and later $Y \rightarrow X$ means $\Sigma^{\infty} Y \rightarrow X$ when $Y$ is a space and $X$ is a spectrum.)

Proof. In the diagram

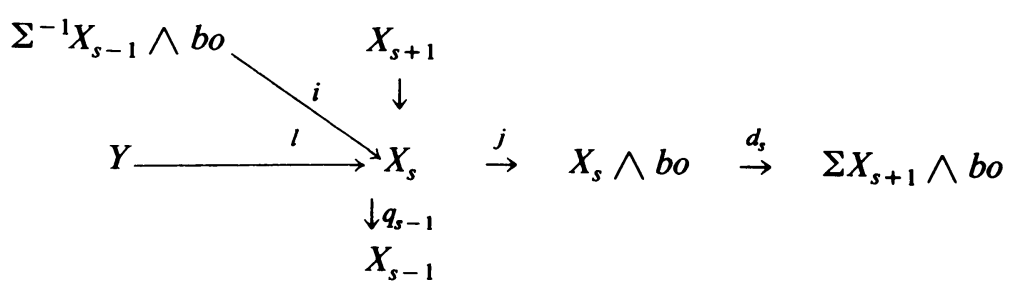

one smash factor of $d_{s} \cdot j$ is $\delta \rightarrow b o \rightarrow \overline{b o}$ so $d_{s} \cdot j$ is trivial. By applying 3.6 to $C_{N}\left(D_{N} Y \wedge X\right)$ and using $S$-duality,

$$
\frac{\operatorname{ker}\left(\left(d_{s}\right)_{*}:\left[Y, X_{s} \wedge b o\right] \rightarrow\left[Y, \Sigma X_{s+1} \wedge b o\right]\right)}{\left.\operatorname{im}(j i)_{*}:\left[Y, \Sigma^{-1} X_{s-1} \wedge b o\right] \rightarrow\left[Y, X_{s} \wedge b o\right]\right)}
$$

is a $\mathbf{Z}_{2}$-vector space all elements of which are detected in $\mathbf{Z}_{2}$-cohomology. Since [jl] $\in \operatorname{ker}\left(d_{s^{*}}\right)$ and $H^{*}(j l)=0$, there exists $\phi: Y \rightarrow \Sigma^{-1} X_{s-1} \wedge$ bo such that $[j i \phi]=$ $[j l]$. Then $l^{\prime}=l-i \phi$ is the desired map.

The remainder of this section will be devoted to the proof of 3.6. It follows quite directly from the methods of [14], but we hope to clarify some aspects of that proof. No aspects of this proof will be required in $\$ 4$.

Let $W$ denote the fiber of $\Omega^{2} S^{3} \rightarrow S^{1}$. Then $\Omega^{2} S^{3} \simeq W \times S^{1}$. The May filtration on $\Omega^{2} S^{3}$ induces a filtration $F_{n}(W)$. Let $\bar{B}(n)$ denote the Thom spectrum defined by the composite $F_{2 n}(W) \hookrightarrow \Omega^{2} S^{3} \rightarrow^{\Omega^{2} g} B O$. Let $B_{n}=\Sigma^{4 n} \bar{B}(n)$ and if $\bar{n}=\left(n_{1}, \ldots, n_{s}\right)$ let $B_{\bar{n}}=B_{n_{1}} \wedge \ldots \wedge B_{n_{s}}$. Let $R_{s}$ denote the set of $s$-tuples of positive integers. If $\left(n_{1}, \ldots, n_{s}\right) \in R_{s}$ and $\varepsilon=0$ or 1 , we say that $\left(n_{1}, \ldots, n_{i+1}, k, n_{i}-k\right.$, $\left.n_{i+1}, \ldots, n_{s}\right)$ is an $\varepsilon$-successor of $\left(n_{1}, \ldots, n_{s}\right)$ if $\nu\left(\begin{array}{c}n_{i} \\ k\end{array}\right)=\varepsilon$. 
A map of spectra $f: X \rightarrow Y$ is a homotopy equivalence $\bmod K\left(\mathbf{Z}_{2}\right)$ 's if there are wedges of $K\left(\mathbf{Z}_{2}\right)$ 's, $K_{1}$ and $K_{2}$, and homotopy equivalences $h_{1}$ and $h_{2}$ so that the $X_{1} \rightarrow Y_{1}$ component of

$$
X_{1} \vee K_{1} \underset{h_{1}}{\stackrel{\simeq}{\rightarrow}} X \underset{f}{\rightarrow} \underset{h_{2}}{\stackrel{\simeq}{\rightarrow}} Y_{1} \vee K_{2}
$$

is a homotopy equivalence. The map of spaces $\Omega^{\infty} X \rightarrow \Omega^{\infty} Y$ is also called a homotopy equivalence $\bmod K\left(\mathbf{Z}_{2}\right)$ 's.

The bulk of the work in proving 3.6 is in

THEOREM 3.7. There are homotopy equivalences

$$
h_{s}: \bigvee_{\bar{n} \in R_{s}} B_{\bar{n}} \wedge b o \rightarrow \overline{b o}^{\wedge s} \wedge b o
$$

such that, for the map $d_{s}$ which induces the chain complexes $C\left(S^{0}\right)$ of 3.6

$$
\bigvee_{\bar{n} \in R_{s}} B_{\bar{n}} \wedge b o \stackrel{h_{s}}{\rightarrow} \overline{b o} \wedge s \wedge \stackrel{d_{s}}{\rightarrow} \overline{b o} \wedge(s+1) \wedge b o \stackrel{h_{s+1}^{-1}}{\rightarrow} \bigvee_{\bar{m} \in R_{s+1}} B_{\bar{m}} \wedge b o,
$$

the component $B_{\bar{n}} \wedge b o \rightarrow B_{\bar{m}} \wedge$ bo

(i) is a homotopy equivalence $\bmod K\left(\mathbf{Z}_{2}\right)$ 's if $\bar{m}$ is a 0 -successor of $\bar{n}$,

(ii) lifts to a homotopy equivalence $\bmod K\left(\mathbf{Z}_{2}\right){ }^{\prime} s B_{\bar{n}} \wedge b o \rightarrow\left(B_{\bar{m}} \wedge b o\right)^{\langle 1\rangle}$ if $\bar{m}$ is a 1-successor of $\bar{n}$,

(iii) has filtration $>1$ if $|\bar{m}|<|\bar{n}|$, or $|\bar{m}|=|\bar{n}|$ and $\bar{m}$ is neither a 0 - nor a 1-successor of $\bar{n}$. (Here and elsewhere $|\bar{n}|=\sum n_{i}$.)

We shall return to the proof of 3.7 at the end of this section. 3.7 is easily adapted to spaces $X$ satisfying the hypotheses of 3.6.

COROLlary 3.8. Suppose the Adams spectral sequences

$$
\operatorname{Ext}_{Q_{1}}\left(H^{*} X, \mathbf{Z}_{2}\right) \Rightarrow \pi_{*}(X \wedge b o)
$$

has no nontrivial differentials. If $X \wedge$ is applied to the maps of 3.7, the component $X \wedge B_{\bar{n}} \wedge$ bo $\rightarrow X \wedge B_{\bar{m}} \wedge$ bo

(i) is a homotopy equivalence $\bmod K\left(\mathbf{Z}_{2}\right)$ 's if $\bar{m}$ is a 0 -successor of $\bar{m}$,

(ii) lifts to a map $X \wedge B_{\bar{n}} \wedge b o \rightarrow\left(X \wedge B_{\bar{m}} \wedge b o\right)^{\langle 1\rangle}$ which is surjective in $\pi_{*}()$ and whose kernel consists at most of filtration zero $\mathbf{Z}_{2}$ 's, if $\bar{m}$ is a 1-successor of $\bar{n}$,

(iii) has filtration $>1$ if $|\bar{m}|<|\bar{n}|$, or $|\bar{m}|=|\bar{n}|$ and $\bar{m}$ is neither a 0 - nor 1-successor of $\bar{n}$.

If there are no differentials through dimension $i$, then the results are true through dimension $i$.

Proof. (i) is clear, and (iii) follows immediately from 3.7(iii) and the map $X \wedge\left(B_{\bar{m}} \wedge b o\right)^{\langle 2\rangle} \rightarrow\left(X \wedge B_{\bar{m}} \wedge b o\right)^{\langle 2\rangle}$. To prove (ii) we use 3.7(ii) to obtain

$$
X \wedge B_{\bar{n}} \wedge b o \stackrel{\simeq}{\rightarrow} X \wedge\left(B_{\bar{m}} \wedge b o\right)^{\langle 1\rangle} \stackrel{l}{\rightarrow}\left(X \wedge B_{\bar{m}} \wedge b o\right)^{\langle 1\rangle} \text {. }
$$

A standard result in homological algebra (e.g. [17, Exercise 3, p. 102]) shows that applying $\operatorname{Ext}_{\mathfrak{Q}}^{s}\left(H^{*}(), \mathbf{Z}_{2}\right)$ to $l$ induces an isomorphism when $s>0$ and an epimorphism when $s=0$. We will be done once we note that the ASS for $X \wedge B_{\bar{n}} \wedge b o$ 
has no nontrivial differentials. This follows from the following lemma which will be proved later in this section.

LEMMA 3.9. If $\bar{n}=\left(n_{1}, \ldots, n_{s}\right)$, let $|\bar{n}|=\sum n_{i}$ and $\alpha(\bar{n})=\sum \alpha\left(n_{i}\right)$.

$$
B_{\bar{n}} \wedge b o \simeq K \vee \begin{cases}\Sigma^{4 \mid \bar{n}} b o^{\langle 2|\bar{n}|-\alpha(\bar{n})\rangle} & \text { if }|\bar{n}| \text { is even, } \\ \Sigma^{4|n|} b s p^{\langle 2|\bar{n}|-1-\alpha(\bar{n})\rangle} & \text { if }|\bar{n}| \text { is odd, }\end{cases}
$$

where $K$ is a wedge of suspensions of $K\left(\mathbf{Z}_{2}\right)$.

Since there is a map $X \wedge\left(b o^{\langle t\rangle}\right) \rightarrow(X \wedge b o)^{\langle t\rangle}$ inducing an isomorphism in $\operatorname{Ext}^{s}()$ for $s>0, X \wedge B_{\bar{n}} \wedge b o$ has no nontrivial differentials when $|\bar{n}|$ is even. A similar argument works when $|\bar{n}|$ is odd once we note that there are no differentials in the ASS for $X \wedge b s p$. This follows from the fact that there are maps $\Sigma^{8} b s p \rightarrow$ $\Sigma^{4} b o \rightarrow b s p$ such that the composite induces an injection in $\operatorname{Ext}_{\mathbb{Q}}\left(H^{*}(X \wedge), \mathbf{Z}_{2}\right)$.

In order to see that 3.8 implies 3.6 , we shall use the following combinatorial result, which orders the summands in $\bigvee_{\bar{n} \in R_{s}} B_{\bar{n}} \wedge$ bo. It may be viewed as an elaboration upon the argument used in [14, proof of 5.11].

LEMMA 3.10. There are partitions $R_{s}=T_{s} \amalg U_{s}$, total orderings $\ll$ on $T_{s}$ and $U_{s}$, and bijections $\Phi: T_{s} \rightarrow U_{s+1}$ so that $\Phi(\bar{n})$ is the first 0-successor of $\bar{n}$ if $\bar{n}$ contains some non-2-powers, and $\phi(\bar{n})$ is the first 1-successor of $\bar{n}$ if $\bar{n}$ consists of all 2-powers. Also, $|\bar{m}|<|\bar{n}|$ implies $\bar{m} \ll \bar{n}$.

We shall return to the rather detailed proof of 3.10 after using it to deduce 3.6.

Proof of 3.6. The case $s=1$ follows from the methods below together with those of [14] and [16], so we shall assume $s \geqslant 2$. The first case of 3.6 follows immediately from the fact that $b o$ is 3-connected.

We may ignore the split $K\left(\mathbf{Z}_{2}\right)$ 's in the equivalences $\bmod K\left(\mathbf{Z}_{2}\right)$ 's of 3.8. We choose homotopy classes $g_{\alpha}$ corresponding to as basis of $\operatorname{Ext}_{\mathscr{Q}}^{0 * *}\left(H^{*}\left(B_{\Phi(\bar{n})} \wedge b o\right)\right)$ for all $\bar{n} \in T_{s-1}$ which consist only of 2-powers. We first show that any element $y$ of $\pi_{*}\left(X \wedge \bar{b} o^{\wedge s} \wedge b o\right)$ plus perhaps some $g_{\alpha}$ 's is equivalent $\bmod \operatorname{im}\left(d_{s-1}\right)$ to an element of $\pi_{*}\left(X \wedge \bigvee_{\bar{n} \in T_{s}} B_{\bar{n}} \wedge b o\right)$. It suffices to show that this is true up to any filtration.

If $z \in \pi_{*}\left(X \wedge \bar{b} o^{\wedge s} \wedge b o\right)$, let $z_{\bar{n}}$ denote the component of $z$ in $\pi_{*}\left(X \wedge B_{\bar{n}} \wedge\right.$ bo). Suppose we have shown that for $\hat{y}=y+d_{s-1}(w)+\sum g_{\alpha}, \operatorname{filtr}\left(\hat{y}_{\bar{n}}\right) \geqslant j$ for all $\bar{n} \in U_{s}$, filtr $\left(\hat{y}_{\bar{n}}\right)>j$ for all $\bar{n} \ll \bar{l}$, and $\operatorname{filtr}\left(\hat{y}_{\bar{l}}\right)=j$. If $j=0$ and $\bar{l}=\phi(\bar{e})$ where $\bar{e}$ has all 2-powers, then by adding an appropriate $g_{\gamma} \in \pi_{*}\left(X \wedge B_{\bar{l}} \wedge b o\right)$, we can make filtr $\left(\hat{y}+g_{\gamma}\right)_{\bar{l}} \geqslant 1$. Otherwise, by 3.10 and 3.8 there exists $v \in \pi_{*}\left(X \wedge B_{\bar{e}} \wedge\right.$ bo) where $l=\phi(\bar{e})$, such that $\left(d_{s-1} v\right)_{\bar{l}}=\hat{y}_{\bar{l}}$. Moreover, we still have

$$
\text { filtr }\left(\hat{y}-d_{s-1} v\right)_{\bar{n}}>j \quad \text { for all } \bar{n} \ll \bar{l}
$$

and

$$
\text { filtr }\left(\hat{y}-d_{s-1} v\right)_{\bar{n}} \geqslant j \quad \text { for all } \bar{n} \in U_{s} .
$$

This inductive procedure enables us to eliminate all $U_{s}$-components. 
We will now show that $d_{s}$ is injective on $\pi_{*}\left(X \wedge \bigvee_{\bar{n} \in T_{s}} B_{\bar{n}} \wedge b o\right)$; by the preceding paragraphs this will imply 3.6. We partition $T_{s}=P_{s} \amalg Q_{s}$, where $P_{s}$ are those tuples consisting of all 2-powers. If $y \neq 0 \in T_{s}$, let

$$
j=\min \left(\left\{\operatorname{filtr}\left(y_{\alpha}\right): \alpha \in Q_{s}\right\} \cup\left\{\operatorname{filtr}\left(y_{\alpha}\right)+1: \alpha \in P_{s}\right\}\right)
$$

and let $\alpha_{0}$ be the smallest $\alpha$ realizing $j$. By 3.10 and 3.8 the $\phi\left(\alpha_{0}\right)$-component of $d_{s} y$ is nonzero.

PROOF OF 3.10. Let $T_{s}=\left\{\left(2^{e_{1}}, \ldots, 2^{e_{s}}\right): e_{1}>e_{2} \geqslant e_{3} \geqslant \cdots \geqslant e_{s}\right\} \cup$ $\left\{\left(n_{1}, \ldots, n_{j-1}, 2^{e_{j}}+k, 2^{e_{j+1}}, \ldots, 2^{e_{s}}\right): 0<k<2^{e_{j}}, e_{j} \geqslant e_{j+1} \geqslant \cdots \geqslant e_{s}\right\}$, $\phi\left(2^{e_{1}}, \ldots, 2^{e_{s}}\right)=\left(2^{e_{1}-1}, 2^{e_{1}-1}, 2^{e_{2}}, \ldots, 2^{e_{s}}\right)$, and

$$
\phi\left(n_{1}, \ldots, n_{j-1}, 2^{e_{j}}+k, 2^{e_{j+1}}, \ldots, 2^{e_{s}}\right)=\left(n_{1}, \ldots, n_{j-1}, k, 2^{e_{j}}, 2^{e_{j+1}}, \ldots, 2^{e_{s}}\right) .
$$

$\phi$ is easily checked to be a bijection $T_{s} \rightarrow R_{s+1}-T_{s+1}$ which preserves ||.

We shall call the elements of $T_{s}$ which contain all 2-powers "type B" and the other elements "type A". A successor is a 0-successor of a type A element or a 1 -successor of a type B element. Note that $\Phi(\bar{n})$ is always a successor of $\bar{n}$. Define a relation $\ll$ on $T_{s}$ by

if $\bar{n} \neq \bar{m} \in T_{s}$ and $\phi(\bar{n})$ is a successor of $\bar{m}$, then $\bar{m} \ll \bar{n}$.

We now show that $(*)$ causes no loops $\bar{m} \ll \bar{n}_{1} \ll \ldots \ll \bar{n}_{q-1} \ll \bar{m}$. Suppose such a loop exists.

Case 1. There are no type B's in the sequence. If $e_{1}<\ldots<e_{r}$, let $p\left(\sum_{i=1}^{r} 2^{e_{i}}\right)=$ $\left(2^{e_{1}}, \ldots, 2^{e_{r}}\right)$ and let $p\left(a_{1}, \ldots, a_{s}\right)=\left(p\left(a_{1}\right), \ldots, p\left(a_{s}\right)\right)$. Then $p(\bar{m})<p\left(\bar{n}_{1}\right)$ $<\ldots<p\left(\bar{n}_{q-1}\right)<p(\bar{m})$ under the lexicographical ordering from the left. But this cannot happen since the lexicographic order is a total order.

Case 2. There is at least one type B. By considering the various type A or B possibilities, one shows that $\bar{m} \ll \bar{n}$ under (*) implies $\nu$ (first component of $\bar{m}$ ) $<$ $\nu$ (first component of $\bar{n}$ ) and if $\bar{n}$ has type $\mathrm{B}$ then this inequality is strict. This implies that there can be no looping under (*) when a type B occurs.

Since there are no loops, « can be extended to a total order on $T_{s}$ (compatible with ||$)$. If $U_{s+1}$ is ordered by $\phi(\ll)$, then $(*)$ guarantees that $\phi(\bar{m})$ is the first successor of $\bar{m}$.

Proof of 3.9. Except for the construction of maps near the end, this argument was essentially given in [14], but again some clarification seems worthwhile.

Let $\operatorname{filtr}\left(\xi_{1}^{i_{1}} \xi_{2}^{i_{2}} \ldots\right)=\sum i_{j} 2^{j-1}$. Then $\chi\left(H^{*} \bar{B}(n)\right)^{*}$ is the subspace $S_{n}$ of $\mathbf{Z}_{2}\left[\xi_{1}^{2}, \xi_{2}, \ldots\right]$ spanned by monomials of filtration $\leqslant 2 n$. This is proved by noting that it is $\operatorname{ker}\left(R_{\mathrm{Sq}^{1}}, R_{\mathrm{Sq}^{2 n+2}}, \ldots: \mathbb{Q}^{*} \rightarrow \bigoplus \mathbb{Q}^{*}\right)$.

For $j \in \mathbf{Z}_{4}$ and $n \geqslant 0$, we define $Q_{1}$-modules $Q_{j, n}$ by induction on $n$ by the nontrivial extension of $\mathbb{Q}_{1}$-modules

$$
0 \rightarrow\left\{1, \mathrm{Sq}^{2}, \mathrm{Sq}^{3}, \mathrm{Sq}^{2} \mathrm{Sq}^{3}\right\} \rightarrow Q_{j, n} \rightarrow \Sigma^{4} Q_{j, n-1} \rightarrow 0
$$

with $Q_{0,0}=\mathbf{Z}_{2}, Q_{1,0}=\left\{1, \mathrm{Sq}^{2}, \mathrm{Sq}^{3}\right\}, Q_{2,0}=\Sigma^{-2}\left\{1, \mathrm{Sq}^{1}, \mathrm{Sq}^{2}, \mathrm{Sq}^{2} \mathrm{Sq}^{1}, \mathrm{Sq}^{3} \mathrm{Sq}^{1}\right\}, Q_{3,0}$ $=\Sigma^{-3}\left\{1, \mathrm{Sq}^{1}, \mathrm{Sq}^{2} \mathrm{Sq}^{1}\right\}$. Note that $Q_{j, n}$ is $P_{i j n} / N_{i}$ in the notation of $[4,3.6]$ (with $j=1$ and 3 reversed) and are the modules $T, S, Y$, and $Z$ of [18, pp. 133-135]. The next lemma is a restatement of $[18,1.15]$. It can also be proved by the method of $[4$, 3.9]. 
LEMMA 3.11. There is an isomorphism of $\mathbb{Q}_{1}$-modules

$$
Q_{j_{1}, n_{1}} \otimes Q_{j_{2}, n_{2}} \approx F \oplus Q_{j_{1}+j_{2}, n_{1}+n_{2}+\delta}
$$

where $F$ is a free $\mathbb{Q}_{1}$-module and

$$
\delta= \begin{cases}-1 & \text { if }\{3\} \subset\left\{j_{1}, j_{2}\right\} \subset\{2,3\} \\ 1 & \text { if }\{1\} \subset\left\{j_{1}, j_{2}\right\} \subset\{1,2\} \\ 0 & \text { otherwise }\end{cases}
$$

LEMMA 3.12. There is an isomorphism of $\mathbb{Q}_{1}$-modules

$$
H^{*}\left(\bar{B}\left(n_{1}\right) \wedge \cdots \wedge \bar{B}\left(n_{s}\right)\right) \approx F \oplus Q_{\Sigma \alpha\left(n_{j}\right), \Sigma n_{j}-D}
$$

where $D=2 l$ if $\sum \alpha\left(n_{j}\right)=4 l$, and $D=2 l+1$ if $4 l+1<\sum \alpha\left(n_{j}\right)<4 l+3$, where $F$ is a free $\mathbb{Q}_{1}$-module.

Proof. The $\mathbb{Q}_{1}$-submodule $T_{2^{i}}$ of $S_{2^{i}}$ generated over $\mathbb{Q}_{1}$ by $\xi_{i+2}$ and $\xi_{j}^{8\left(2^{i-1-j}-1-k\right)} \xi_{j+1}^{4 k+2} \xi_{j+2}, 0 \leqslant k \leqslant 2^{i-1-j}-1,0 \leqslant j \leqslant i-1$, has its dual isomorphic to $Q_{1,2^{i}-1}$. Multiplying out $\bigotimes_{2^{i} \in n} T_{2^{i}} \rightarrow S_{n}$ induces an isomorphism in $Q_{0^{-}}$and $Q_{1}$-homology. The result now follows from several applications of 3.11 .

The following result is our restatement of $[18,2.4]$.

LEMMA 3.13. There are isomorphisms of $\mathbb{Q}$-modules

$$
\begin{aligned}
H^{*}\left(b o^{\langle n\rangle}\right) & \approx \mathbb{Q} \otimes_{\mathcal{Q}_{1}} Q_{-n,\left(n+\varepsilon_{1}\right) / 2} \\
H^{*}(b s p & \langle n\rangle)
\end{aligned}
$$

where

$$
\varepsilon_{1}= \begin{cases}1, & n \equiv 1(4) \\ -1, & n \equiv 3(4), \\ 0, & n \text { even }\end{cases}
$$

and

$$
\varepsilon_{2}= \begin{cases}2, & n \equiv 2(4) \\ 0, & n \equiv 0(4) \\ 1, & n \text { odd }\end{cases}
$$

3.12 and 3.13 imply that the spaces in 3.9 have isomorphic cohomology modules. It remains to construct the maps realizing the isomorphisms. The main step is the $\operatorname{map} \bar{B}\left(2^{i}\right) \rightarrow b o^{\left\langle 2^{i+1}-1\right\rangle}$.

By the Adams edge theorem [1], a chart for the ASS of $\mathfrak{S}^{\left\langle 2^{i+1}-1\right\rangle}$ begins

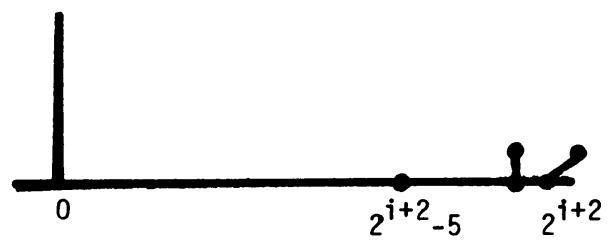


Thus the inclusion of the bottom cell of $\bar{B}\left(2^{i}\right)$ into $\varsigma^{\left\langle 2^{i+1}-1\right\rangle}$ extends over the $\left(2^{i+2}-5\right)$-skeleton. The attaching map for the next cell has positive filtration in $\pi_{2^{i+2}-5}\left(\bar{B}\left(2^{i}\right)^{\left(2^{i+2}-5\right)}\right)$ and hence maps to 0 in $\pi_{2^{i+2}-5}\left(\varsigma^{\left\langle 2^{i+1}-1\right\rangle}\right)$. Since $H^{j}\left(\bar{B}\left(2^{i}\right)\right)=0$ for $j \geqslant 2^{i+2}$, there are no obstructions to extending the map to $\bar{B}\left(2^{i}\right) \rightarrow \mathcal{S}^{\left\langle 2^{i+1}-1\right\rangle}$. Following into $b o^{\left\langle 2^{i+1}-1\right\rangle}$ yields our desired map.

After applying $\wedge b o$, the map $\bigwedge_{2^{i} \in n} \bar{B}\left(2^{i}\right) \rightarrow \bar{B}(n)$ discussed in [12] and [14] becomes a homotopy equivalence $\bmod K\left(\mathbf{Z}_{2}\right)$ 's. (This follows from 3.12 or from [14].) A homotopy inverse on the irreducible part followed by the smash product of the maps constructed above and the map $\bigwedge_{2^{i} \in n} b o^{\left\langle 2^{i+1}-1\right\rangle} \rightarrow b o^{\langle 2 n-\alpha(n)\rangle}$ yields the desired map $\bar{B}(n) \rightarrow b o^{\langle 2 n-\alpha(n)\rangle}$ if $n$ is even. The case $n$ odd and the case of products of $\bar{B}(n)$ 's follow from these methods using also the maps $b s p \wedge b o \rightarrow b s p$ and $b s p \wedge b s p \rightarrow b o^{\langle 2\rangle}$.

We now proceed toward the proof of 3.7, the final piece of unfinished business.

LEMMA 3.14. There is an isomorphism of $\mathbb{Q}_{1}$-modules

$$
\bigoplus_{n>0} \chi H_{*} B_{n} \stackrel{\bigoplus x f_{n^{*}}}{\rightarrow} \chi H_{*} \text { bo }
$$

given by $\chi f_{n^{*}}\left(\xi_{1}^{i_{1}} \xi_{2}^{i_{2}} \ldots\right)=\xi_{1}^{4 n-\Sigma 2^{i_{i}}} \xi_{2}^{i_{1}} \xi_{3}^{i_{2}} \ldots$

Proof. By [2], $\chi H_{*} b o=\mathbf{Z}_{2}\left[\xi_{1}^{4}, \xi_{2}^{2}, \xi_{3}, \ldots\right] \cdot \chi f_{n^{*}}$ is an $\mathbb{Q}_{1}$-morphism, injective, and onto the filtration $4 n$ part of $\chi H_{*} b o$.

LEMMA 3.15. Let $m=(\mu \wedge \mu) \circ(1 \wedge T \wedge 1):(b o \wedge b o) \wedge(b o \wedge b o) \rightarrow b o \wedge$ bo. Let $\Phi_{n, m}: B_{n} \wedge B_{m} \rightarrow B_{n+m}$ denote the multiplication. There are maps $f_{n}: B_{n} \rightarrow$ bo $\wedge$ bo such that $f_{n^{*}}\left(i_{n}\right)=\xi_{1}^{4 n} \otimes 1$, and if $n=2^{i}+k, 1<k \leqslant 2^{i}$, then $f_{n} \circ \phi_{2^{i}, k}-$ $m \circ\left(f_{2^{i}} \wedge f_{k}\right)$ has filtration $\geqslant 4$.

Proof. The maps will be constructed inductively to have the multiplicative property. The homology property will follow automatically. Indeed we will have $\chi f_{n^{*}}\left(\xi_{1}^{i_{1}} \xi_{2}^{i_{2}} \ldots\right)=\xi_{1}^{4 n-\Sigma 2^{i} j_{j}} \xi_{2}^{i_{1}} \xi_{3}^{i_{2}} \ldots \otimes 1$.

If $k<2^{i}$ the element of filtration $\geqslant 4$ is not required in the multiplicative property. We show that

$$
\left[m\left(f_{2^{i}} \wedge f_{k}\right)\right] \in \operatorname{im}\left(\phi_{2^{i}, k}^{*}:\left[B_{2^{i}+k}, b o \wedge b o\right] \rightarrow\left[B_{2^{i}} \wedge B_{k}, b o \wedge b o\right]\right) .
$$

These groups are calculated from the ASS where $E_{2}$-terms are

$$
\left.\operatorname{Ext}_{\mathscr{Q}_{1}}^{s, *}\left(\mathscr{Q} / / \mathbb{Q}_{1}, H^{*} B_{2^{i}+k}\right) \rightarrow \operatorname{Ext}_{\mathscr{Q}_{1}^{s *}}^{s, Q} / / \mathbb{Q}_{1}, H^{*}\left(B_{2^{i}} \wedge B_{k}\right)\right) \text {. }
$$

By 3.12 these homomorphisms fit into a long exact sequence with $\operatorname{Ext}_{\mathbb{Q}_{1}}\left(\mathscr{Q} / / \mathbb{Q}_{1}\right.$, free $\mathbb{Q}_{1}$-module), and hence are isomorphisms for $s>0$. Thus it suffices to show that the cohomology homomorphism $\left(m\left(f_{2^{i}} \wedge f_{k}\right)\right)^{*} \in \operatorname{im}\left(\phi_{2^{i}, k}^{*}\right)$, or equivalently $\chi\left(m\left(f_{2^{i}} \wedge f_{k}\right)\right)_{*}(K)=0$, where $K=\operatorname{ker}\left(\chi\left(\phi_{2^{i}, k}\right)_{*}\right)$. But $K$ is a free $Q_{1}$-module spanned over $\mathbf{Z}_{2}$ by $\xi^{I_{1}} \otimes \xi^{J_{1}}+\xi^{I_{2}} \otimes \xi^{J_{2}}$ with $I_{1}+J_{1}=I_{2}+J_{2}$, which is annihilated by the multiplication map $\chi\left(\phi_{2^{i}, k}\right)_{*}$.

For the case $k=2^{i}$, we will need the following.

Proposition 3.16. Let $F_{i}=\Sigma^{2^{i+4}-5} M_{2} \wedge \bar{B}(1)$. There is a map $F_{i} \rightarrow^{j} B_{2^{i}} \wedge B_{2^{i}}$ whose cofiber is equivalent $\bmod K\left(\mathbf{Z}_{2}\right)$ 's to $B_{2^{i+1}}$. 
Proof. By $3.12, \phi_{2^{i}, 2^{i}}$ is a $\left(2^{i+4}-5\right)$-equivalence, and the homotopy charts are
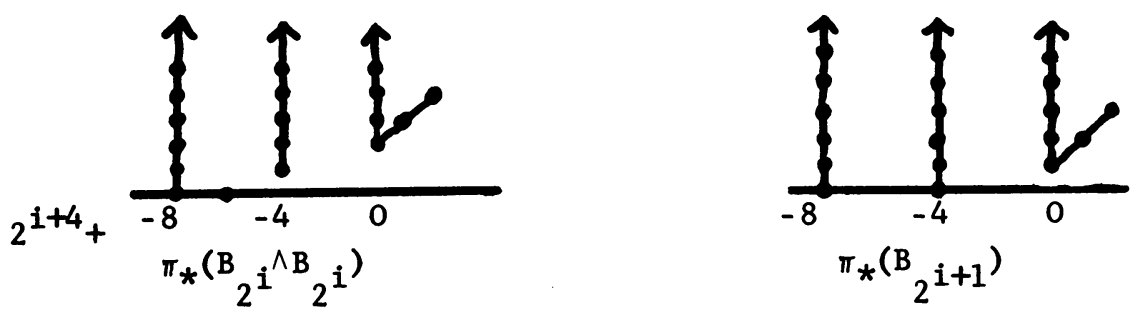

There are no obstructions to extending the map $\Sigma^{2^{i+4}-5} M \rightarrow{ }^{p} S^{2^{i+4}-4} \rightarrow^{g} B_{2^{i}} \wedge B_{2^{i}}$ over $F_{i}$. The composite $F_{i} \rightarrow^{j} B_{2^{i}} \wedge B_{2^{i}} \rightarrow B_{2^{i+1}}$ is trivial, so that there exists $M C(j) \rightarrow B_{2^{i+1}}$, which is easily seen to induce an isomorphism above filtration zero. Indeed the chart for $\pi_{*}(M C(j))$ is obtained from those of $B_{2^{i}} \wedge B_{2^{i}}$ and $F$ as below:

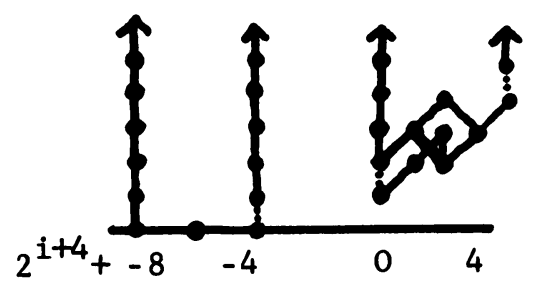

3.15 now follows immediately from the following calculation.

LEMMA 3.17. If $m \in\left[B_{2^{i}} \wedge B_{2^{i}}\right.$, bo $\wedge$ bo] satisfies $m_{*}\left(i_{2^{i}} \otimes i_{2^{i}}\right)=\xi_{1}^{i^{i+3}}$ there exists $q$ of filtration $\geqslant i+4$ such that $j^{*}(m+q)=0 \in\left[F_{i}\right.$, bo $\wedge$ bo $]$.

Proof. From 3.14 and $3.9, \pi_{*}\left(b o \wedge b o\right.$ ) in the range $2^{i+4}-8<*<2^{i+4}+2$ is a sum of charts of the following three types:

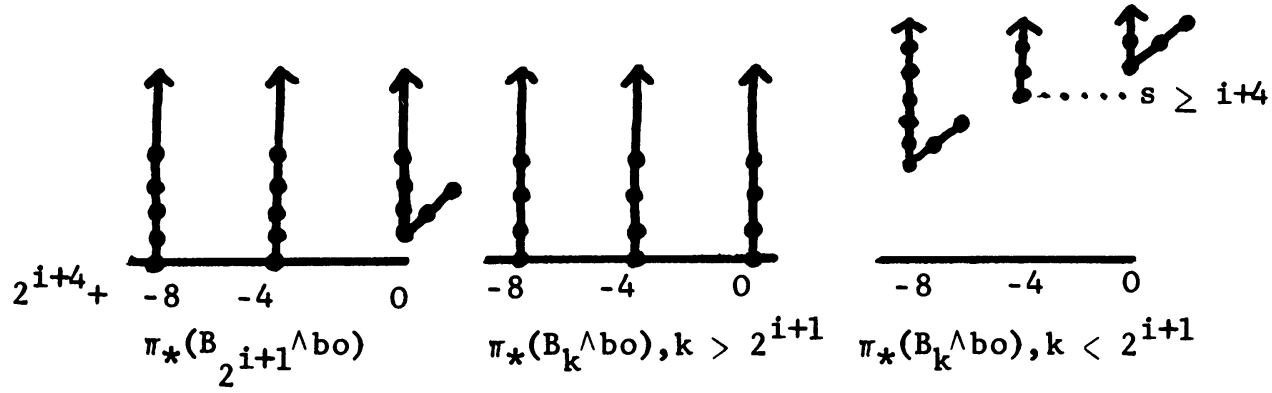

Above filtration zero $\left[F_{i}, b o \wedge b o\right] \approx \pi_{2^{i+4}-4}(b o \wedge b o) \otimes \mathbf{Z}_{2}$. Since $j^{*} m$ has positive filtration, it corresponds to some of the $\mathrm{Z}_{2}$ 's in filtration $>i+4$ (or else is zero). Since we may assume by induction that $b o \wedge b o$ has been split through a range into $\bigvee B_{k} \wedge b o$ (or by just looking at the splitting of Ext-groups), it suffices to show:

if $k<2^{i+1}$ and the generator of the $\mathbf{Z}$ in $\pi_{2^{1+4}-4}\left(B_{k} \wedge b o\right)$ has filtration $l$, then $\left[B_{2^{i}} \wedge B_{2^{i}}, B_{k} \wedge b o\right]$ contains a filtration $l$ class $f$ such that $j^{*} f \neq 0 \in\left[F_{i}, B_{k} \wedge b o\right]$. 
This may be verified by direct calculation of Ext-groups or (as in [14]) by letting $f$ be the restriction of the usual map

$$
\begin{array}{ccc}
\Sigma^{2^{i+3}} b o^{\left\langle 2^{i+2}-2\right\rangle} & \rightarrow & \Sigma^{2^{i+3}} b o \\
\Sigma^{2^{i+3}} B_{2^{i}} \wedge B_{2^{i}} \wedge b o & & \left(B_{k} \wedge b o\right)\left[2^{i+3}\right]
\end{array}
$$

where the first equality is by 3.9 and the second is easily proved by starting with the map $S^{2^{i+3}} \rightarrow B_{k} \wedge$ bo corresponding to a homotopy generator.

Now we can prove the next result, which is a major step toward 3.7. Let $\Omega S_{+}^{5} \wedge X_{5} \rightarrow^{h} X_{5} \wedge X_{5}$ be the homotopy equivalence constructed in [15]. Let $o:$ $X_{5} \rightarrow b o$ be the rational equivalence given by the bo-orientation. Let $g: \bigvee S^{4 i} \rightarrow$ $\Omega S^{5}$ be a fixed stable equivalence.

THEOREM 3.18. There is a homotopy equivalence $f$ such that the diagram

$$
\begin{array}{ccc}
\bigvee S^{4 n} \wedge X_{5} & \stackrel{g \wedge 1}{\rightarrow} & \Omega S_{+}^{5} \wedge X_{5} \\
j \downarrow \wedge o & & \downarrow(o \wedge o) h \\
\bigvee B_{n} \wedge b o & \stackrel{f}{\rightarrow} & b o \wedge b o
\end{array}
$$

commutes modulo elements of filtration $\geqslant 4$, where $j$ is the inclusion of the bottom cell.

Proof. Suppose $n=2^{i}+k, 0<k \leqslant 2^{i}$. In the diagram

$$
\begin{array}{ccccc}
S^{4 \cdot 2^{i}} & \wedge S^{4 k} & \rightarrow & \left(\Omega S_{+}^{5} \wedge X_{5}\right) \wedge\left(\Omega S_{+}^{5} \wedge X_{5}\right) \stackrel{(\mu \wedge \mu) \circ(1 \wedge T \wedge 1)}{\longrightarrow} \Omega S_{+}^{5} \wedge X_{5} \\
\downarrow j & & (o \wedge o) h \downarrow(o \wedge o) h & \downarrow(o \wedge o) h \\
B_{2^{i}} & \wedge B_{k} & \stackrel{f_{z^{i}} \wedge f_{k}}{\rightarrow} & (b o \wedge b o) \wedge(b o \wedge b o) \stackrel{(\mu \wedge \mu) \circ(1 \wedge T \wedge 1)}{\longrightarrow} \text { bo } \wedge \text { bo }
\end{array}
$$

where the unlabeled arrow ulitizes $g$ and the inclusion of the bottom cell of $X_{5}$, and $f_{2^{i}}$ is the map constructed in 3.15 , the right-hand square does not commute, but does when restricted to the bottom cell of $X_{5}$. (Look at the map of base spaces underlying $h$.) The left-hand square commutes up to elements of filtration $\geqslant 4$ by induction, and hence by the construction of $f_{n}$, we deduce that

$$
\begin{array}{ccc}
S^{4 n} & \stackrel{i}{\rightarrow} & \Omega S_{+}^{5} \wedge X_{5} \\
j \downarrow & & \downarrow \\
B_{n} & \stackrel{f_{n}}{\rightarrow} & b o \wedge b o
\end{array}
$$

commutes up to elements of filtration 4.

Hence in the diagram

$$
\begin{array}{ccccc}
S^{4 n} \wedge X_{5} & \stackrel{i \wedge 1}{\rightarrow} & \Omega S_{+}^{5} \wedge X_{5} \wedge X_{5} & \stackrel{1 \wedge \mu}{\rightarrow} & \Omega S_{+}^{5} \wedge X_{5} \\
j \wedge o \downarrow & & \downarrow(o \wedge o) h) \wedge o & & \downarrow(o \wedge o) h \\
B_{n} \wedge b o & \stackrel{f_{n} \wedge 1}{\rightarrow} & b o \wedge b o \wedge b o & \stackrel{1 \wedge \mu}{\rightarrow} & b o \wedge b o
\end{array}
$$


the left square commutes up to elements of filtration $>4$, and the right square commutes (because the map $\Omega S^{5} \times \Omega S^{5} \rightarrow^{q} \Omega S^{5} \times \Omega S^{5}$ underlying $h^{-1}$ sends $(x, y) \rightarrow\left(x, x^{-1} y\right)$ thus satisfying $\left.(1 \times m)(q \times 1)=q(1 \times m)\right)$.

$f=\bigvee(1 \wedge \mu)\left(f_{n} \wedge 1\right)$ is a homotopy equivalence by 3.14 .

The homotopy equivalences of 3.7 are obtained by iterating 3.18. For example, $h_{2}$ is obtained as

$$
\left(\bigvee B_{n}\right) \wedge\left(\bigvee B_{n}\right) \wedge b o \stackrel{1 \wedge f}{\rightarrow}\left(\bigvee B_{n}\right) \wedge b o \wedge b o \stackrel{f \wedge 1}{\rightarrow} b o \wedge b o \wedge b o \stackrel{1 \wedge \mu}{\rightarrow} b o \wedge b o
$$

It is easy to deduce from 3.18 that

$$
\begin{array}{cccc}
\bigvee S^{4 \bar{n}} \wedge X_{5}= & \bigvee S^{4 n_{1}} \wedge \ldots \wedge \bigvee S^{4 n_{3}} \wedge X_{5} & \rightarrow & \left(\Omega S_{+}^{5}\right)^{\wedge s} \wedge X_{5} \\
j_{1} \wedge \ldots \wedge j_{s} \downarrow \wedge o & \downarrow \\
\bigvee B_{\bar{n}} \wedge b o & =\quad \bigvee B_{n_{1}} \wedge \ldots \wedge \bigvee B_{n_{s}} \wedge b o & \rightarrow & (b o)^{\wedge s} \wedge b o
\end{array}
$$

commutes modulo elements of filtration $\geqslant 4$.

We shall use $X_{5}$ to deduce information about the map $d_{s}$ of 3.7 by the diagram

$$
\begin{aligned}
& \rightarrow\left(\Omega S^{5}\right)^{\wedge s} \wedge X_{5} \rightarrow\left(\Omega S^{5}\right)^{\wedge(s+1)} \wedge X_{5} \rightarrow \\
& \rightarrow\left(\bar{X}_{5}\right)^{\wedge s} \wedge X_{5} \stackrel{1 \wedge p \wedge i}{\rightarrow}\left(\bar{X}_{5}\right)^{\wedge(s+1)} \wedge X_{5} \quad \rightarrow
\end{aligned}
$$

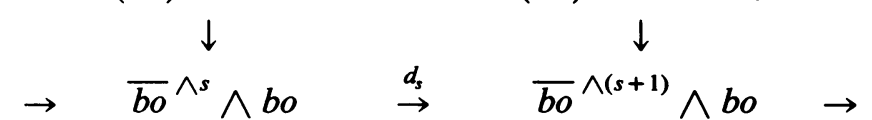

$$
\begin{aligned}
& h_{s} \uparrow \simeq \quad h_{s+1} \uparrow \simeq \\
& \bigvee_{\bar{n} \in R_{s}} B_{\bar{n}} \wedge \text { bo } \quad \bigvee_{\bar{m} \in R_{s+1}} B_{\bar{m}} \wedge b o
\end{aligned}
$$

where the first row is the alternating sum of the diagonals. The first square commutes by $[15,3.8]$ or $[14,5.8]$. By the remarks following 3.18 the diagram is equivalent to

$$
\begin{array}{ccc}
\bigvee_{\bar{n} \in R_{s}} S^{\bar{n}} \wedge X_{5} & \rightarrow & \bigvee_{\bar{m} \in R_{s+1}} S^{\bar{m}} \wedge X_{5} \\
\downarrow & & \downarrow j \wedge o \\
\bigvee_{\bar{n} \in R_{s}} B_{\bar{n}} \wedge b o & \rightarrow & \bigvee_{\bar{m} \in R_{s+1}} B_{\bar{m}} \wedge b o
\end{array}
$$

where the map in homology induced by the top map is

$$
\begin{aligned}
x^{i_{1}} \otimes \cdots \otimes & x^{i_{s+1}} \\
& \mapsto \sum_{j} \sum_{a}(-1)^{y}\left(\begin{array}{l}
i_{j} \\
a
\end{array}\right) x^{i_{1}} \otimes \cdots \otimes x^{i_{j-1}} \otimes x^{a} \otimes x^{i_{j}-a} \otimes \cdots \otimes x^{i_{s+1}} .
\end{aligned}
$$

Proof of 3.7(i). If $\bar{m}$ is a 0 -successor of $\bar{n}$, the map $S^{\bar{n}} \wedge X_{5} \rightarrow S^{\bar{m}} \wedge X_{5}$ is a 2-equivalence. The map of $Q_{0}$-homology induced by $(j \wedge o)^{*}: H^{*}\left(B_{\bar{n}} \wedge b o\right) \rightarrow$ $H^{*}\left(S^{\bar{n}} \wedge X_{5}\right)$ is an isomorphism since the first is spanned by $i_{\bar{n}} \otimes \chi \mathrm{Sq}^{4 i}, i>0$. Thus the infinite towers in $\pi_{4 i}\left(S^{\bar{n}} \wedge X_{5}\right)$ are mapped in a filtration-preserving way to $\pi_{4 i}\left(B_{\bar{n}} \wedge b o\right)$. Filling in the diagram, $\pi_{4 i}\left(B_{\bar{n}} \wedge b o\right) \rightarrow \pi_{4 i}\left(B_{\bar{m}} \wedge b o\right)$ must be 
filtration preserving. By 3.9 or $3.12, B_{\bar{n}} \wedge$ bo and $B_{\bar{m}} \wedge$ bo have isomorphic homotopy charts above filtration 0 of the form

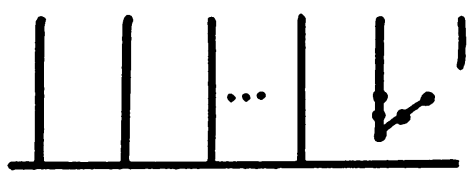

where the precise way in which the towers leave filtration 0 depends upon the mod 4 values of $\alpha(\bar{n})$.

3.7(ii) follows similarly from the observations that if $\bar{m}$ is a 1-successor of $\bar{n}$, then

(1) the homotopy charts of $B_{\bar{n}} \wedge b o$ and $B_{\bar{m}} \wedge$ bo are isomorphic (above filtration 0 ) until they leave filtration 0 , at which point the towers of $B_{\bar{m}} \wedge$ bo always begin one level higher than those of $B_{\bar{n}} \wedge b o$; for example:
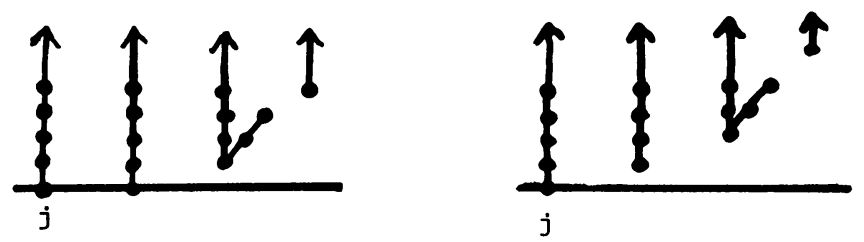

(2) the map $S^{\bar{n}} \wedge X_{5} \rightarrow S^{\bar{m}} \wedge X_{5}$ lifts to a map $S^{\bar{n}} \wedge X_{5} \rightarrow S^{\bar{m}} \wedge X_{5}^{\langle 1\rangle}$ which induces an isomorphism in $H_{*}\left(H^{*}() ; Q_{0}\right)$ and hence of infinite homotopy towers. $\left[H^{*}\left(\Sigma X_{5}^{\langle 1\rangle}\right)=\operatorname{ker}\left(Q \rightarrow\left\{\chi \mathrm{Sq}^{4 i}\right\}\right)\right.$ so that $H_{*}\left(H^{*}\left(\Sigma X_{5}^{\langle 1\rangle}\right) ; Q_{0}\right)$ is spanned by $\left\{\mathrm{Sq}^{1}\right.$. $\left.\chi \mathrm{Sq}^{4 i}\right\} . H^{*}\left(X_{5}^{\langle 1\rangle}\right) \rightarrow H^{*}\left(X_{5}\right)$ sends $\mathrm{Sq}^{1} \rightarrow i$ and hence $\mathrm{Sq}^{1} \cdot \chi \mathrm{Sq}^{4 i}=\chi \mathrm{Sq}^{4 i} \cdot \mathrm{Sq}^{1}+$ $\chi \mathrm{Sq}^{4 i-1} \cdot \mathrm{Sq}^{2} \mapsto \chi \mathrm{Sq}^{4 i}$.]

3.7(iii) follows similarly because if $|\bar{n}|=|\bar{m}|$ and $B_{\bar{n}} \wedge b o \rightarrow B_{\bar{m}} \wedge$ bo has filtration 0 or 1 , then one of the infinite homotopy towers must be mapped by filtration 0 or 1 , and hence the same must be true of $S^{\bar{n}} \wedge X_{5} \rightarrow S^{\bar{m}} \wedge X_{5}$. But the above analysis shows this cannot happen unless $\bar{m}$ is a 0 - or 1-successor of $\bar{n}$. For example, if $H^{*}\left(X_{5}^{\langle 1\rangle}\right) \rightarrow H^{*}\left(X_{5}\right)$ sends $\mathrm{Sq}^{1} \rightarrow 0$, then all elements of $H_{*}\left(H^{*}\left(X_{5}^{\langle 1\rangle}\right) ; Q_{0}\right)$ are mapped trivially. If $|\bar{m}|<|\bar{n}|$ any $\mathbb{Q}$-homomorphism $H^{*}\left(S^{\bar{m}} \wedge X_{5}\right) \rightarrow H^{*}\left(S^{\bar{n}} \wedge X_{5}\right)$ must be 0 , and similarly for the $Q_{0}$-homology elements in $H^{*}\left(S^{\bar{m}} \wedge X_{5}^{\langle 1\rangle}\right)$.

4. Lifting results. In this section we prove the lifting part of Theorem 1.1. We will use the maps $\phi: P_{k+8}^{l+8} \rightarrow P_{k}^{l}$ mentioned in the introduction. They were first constructed in [8] and studied in more detail in [10]. Because $\phi$ is defined only when $l$ is even, Theorem 1.1 is somewhat more difficult to prove when $n$ is odd; the case $n$ odd will be considered in Remark 4.4. 
The lifting part of Theorem 1.1 when $n \equiv 2(8)$ and $e \equiv 0$ (4) is an immediate consequence of

THEOREM 4.1. If $e \equiv 0$ (4) and $e \geqslant 20$ and f classifies $a \cdot 2^{e+6} \xi$ with $a$ odd, then for some $d \leqslant 1+\left[\log _{2}(e+2)\right] / 2$ the composite

$$
\Sigma^{-1} P_{2 e+11+8 d}^{4 e+10+8 d} \stackrel{\phi^{d}}{\rightarrow} \Sigma^{-1} P_{2 e+11}^{4 e+10} \stackrel{f}{\rightarrow} S O \rightarrow V_{2 e+1}
$$

is null-homotopic.

The other cases of Theorem 1.1 when $n$ is even follow from similar results with slight changes in the parameters. (See Remark 4.3.) The parameters here had to satisfy $2^{e} \cdot a 2^{e+6} \xi_{4 e+10}$ trivial, $a 2^{e+6} \xi_{2 e+10}$ trivial, and $(4 e+10)+8<2(2 e+11)$ -1 .

Proof of 4.1. Let $t=e+6-\left[\log _{2}(4 e+10)\right]$. By 3.4, $f$ lifts to $S O^{\langle t\rangle}$, and we follow into $V_{2 e+1}^{\langle t\rangle}$. By 3.3 if $4 e+10+8 d \leqslant 2(2 e+1)+2 t-4$, it will suffice to show $\Sigma^{-1} P_{2 e+11+8 d}^{4 e+10+8 d} \rightarrow{ }^{d} \Sigma^{-1} P_{2 e+11}^{4 e+10} \rightarrow^{f^{\prime}} P_{2 e+1}^{\langle t\rangle}=X$ is null-homotopic. Since $X=$ $P_{2 e+1}^{\langle t\rangle}$ is $\Omega^{\infty}$ of a stable Adams resolution, it suffices to show the stable map $\Sigma^{\infty}\left(f^{\prime} \phi^{d}\right)$ is null-homotopic. The condition simplifies to

$$
4 d \leqslant e-\left[\log _{2}(4 e+10)\right] \text {. }
$$

By the Adams edge theorem [1] for $P_{2 e+1}, X$ is at least $(2 e-2+2 t)$-connected. If $X_{s}$ denotes the $s$ th stage in the $b o$-resolution of $X$, then $X_{s}$ is at least $(2 e-2+2 t$ $+3 s$ )-connected.

We shall return later to the proof of

LEMMA 4.2. If $l$ is even, $k$ odd, and $l+8<2 k-1, \phi: P_{k+8}^{l+8} \rightarrow P_{k}^{l}$ can be written as a composite

$$
P_{k+8}^{l+8}=Y_{4} \stackrel{g_{4}}{\rightarrow} Y_{3} \stackrel{g_{3}}{\rightarrow} Y_{2} \stackrel{g_{2}}{\rightarrow} Y_{1} \stackrel{g_{1}}{\rightarrow} Y_{0}=P_{k}^{l}
$$

such that

(i) $H^{*}\left(g_{i} ; \mathbf{Z}_{2}\right)=0$ for $1 \leqslant i \leqslant 4$.

(ii) For $0 \leqslant i \leqslant 4$ and $X$ as above, the ASS for $D_{N} Y_{i} \wedge X \wedge$ bo has no nontrivial differentials through dimension $N$.

In the diagram

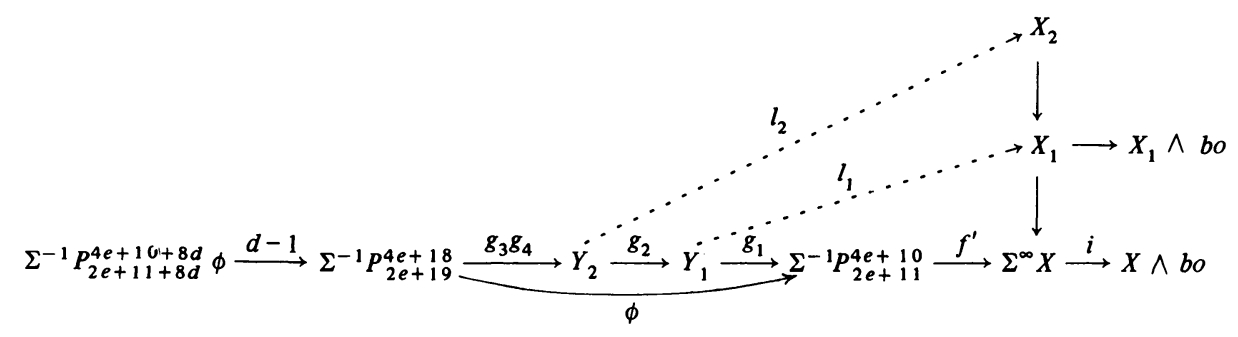


where $g_{1}$ and $g_{2}$ are as in 4.2, we shall show in the next paragraph that the liftings $l_{1}$ and $l_{2}$ exist. Then 4.2 and 3.6.1 imply that $f^{\prime} \circ \phi^{d}$ lifts to $X_{4 d}$, which is at least $(2 e-2+2 t+12 d)$-connected. If $4 d \geqslant 2\left[\log _{2}(4 e+10)\right]-1$, this connectivity is $\geqslant 4 e+9+8 d$, and so the map $\Sigma^{-1} P_{2 e+11+8 d}^{4 e+10+8 d} \rightarrow X_{4 d}$ is trivial, and hence so is $f^{\prime} \circ \phi^{d}$. Comparing with (*), such a $d$ can be chosen if $4 d$ can be chosen in the interval $\left[2\left[\log _{2}(4 e+10)\right]-1, e-\left[\log _{2}(4 e+10)\right]\right]$, and thus can be done if $e>20$.

The proof of 4.1 is completed by showing the existence of $l_{1}$ and $l_{2}$ above. $l_{1}$ follows from the observation that any map $Y_{1} \rightarrow X \wedge$ bo which is cohomologically trivial is null-homotopic. [An associated graded for $\left[Y_{1}, X \wedge b o\right]$ is given by $\operatorname{Ext}_{\mathscr{Q}_{1}}^{s, s}\left(H^{*} X, H^{*} Y_{1}\right)$. By the proof below of 4.2, $D Y_{1}=\left(D\left(\Sigma^{-1} P_{2 e+19}^{4 e+18}\right)\right)^{\langle 3\rangle}$ through a sufficiently large skeleton. Thus for $s>0$,

$$
\operatorname{Ext}_{\mathbb{Q}_{1}}^{s, s}\left(H^{*} X, H^{*} Y_{1}\right) \approx \operatorname{Ext}_{\mathscr{Q}_{1}}^{s+t-3, s+t-3}\left(H^{*} P_{2 e+1}, H^{*}\left(\Sigma^{-1} P_{2 e+19}^{4 e+18}\right)\right)=0
$$

by $[6,3.4($ iii) $]$.]

By 3.7 and $3.9 X_{1} \wedge$ bo splits as $\left(X \wedge \Sigma^{3} b s p\right) \vee W$, and by 3.6 (and the argument in 3.6.1), since the map $Y_{2} \rightarrow X_{1} \wedge$ bo is cohomologically trivial, $l_{1}$ can be varied (if necessary) through $\Omega(X \wedge b o)$ so that the component into $W$ is trivial. A proof similar to that of the previous paragraph shows

$$
\left[Y_{2}, X \wedge \Sigma^{3} b s p\right] \stackrel{\left(g_{3} g_{4}\right)^{*} q_{*}}{\rightarrow}\left[\Sigma^{-1} P_{2 e+19}^{4 e+18}, P_{2 e+1} \wedge \Sigma^{3} b s p\right]
$$

is injective on cohomologically trivial maps, where $q$ is the usual map $X=P_{2 e+1}^{\langle t\rangle} \rightarrow$ $P_{2 e+1} \cdot\left(g_{3} g_{4}\right)^{*} q_{*}\left(l_{1}\right)$ is a map which when followed into $P_{2 e+1} \wedge b J$ is

$$
\Sigma^{-1} P_{2 e+19}^{4 e+18} \rightarrow^{\phi} \Sigma^{-1} P_{2 e+11}^{4 e+10} \rightarrow^{f} S O \rightarrow \Omega^{\infty}\left(P_{2 e+1} \wedge b J\right)
$$

which is trivial since we have chosen these cases so that the $b J$-primary obstructions considered in $\$ 2$ are zero. Since

$$
\left[\Sigma^{-1} P_{2 e+19}^{4 e+18}, P_{2 e+1} \wedge \Sigma^{3} b s p\right] \rightarrow\left[\Sigma^{-1} P_{2 e+19}^{4 e+18}, P_{2 e+1} \wedge b J\right]
$$

is injective above filtration 0 , this implies that our map $Y_{2} \rightarrow X_{1} \wedge$ bo is trivial.

REMARK 4.3. In order to prove the analogue of 4.1 for some of the other congruences it is necessary to use the precise upper edge for $\operatorname{Ext}_{\mathscr{Q}}\left(H^{*} P_{k}, \mathbf{Z}_{2}\right)$ rather than the linear approximation to it used in 3.3 and in the estimate of the connectivity of $X$ in the proof of 4.1 (see Remark 3.3.1). Let $D(\alpha, \beta)$ denote the smallest $j>\alpha$ such that $\pi_{j}\left(P_{\alpha}\right)$ has a nonzero homotopy class of filtration $\beta$. Then $D(\alpha, \beta)=\alpha+2 \beta-\varepsilon(\alpha, \beta)$ where

\begin{tabular}{cc|cccc}
\multicolumn{1}{c}{$\varepsilon(\alpha, \beta)$} & \multicolumn{4}{c}{$\beta(\bmod 4)$} \\
& $\searrow$ & 0 & 1 & 2 & 3 \\
\cline { 3 - 6 }$\alpha(\bmod 4)$ & 0 & 1 & 1 & 2 & 3 \\
& 1 & 0 & 1 & 2 & 0 \\
& 2 & 1 & 1 & 1 & 1 \\
& 3 & 0 & 0 & 1 & 2
\end{tabular}


If we are trying to show $\Sigma^{-1} P_{2 e+c+8 d}^{4 e+b+8 d} \rightarrow^{\phi^{d}} \Sigma^{-1} P_{2 e+c}^{4 e+b} \rightarrow^{2^{e+a} \xi} V_{2 e+\delta}^{\langle t\rangle}$ is null-homotopic, where $t=e+a-\left[\log _{2}(4 e+b)\right]$, then the two conditions on $d$ are

$$
\begin{gathered}
4 e+b-1+8 d<D(2 e+\delta, t)+2 e+\delta-1, \\
D(2 e+\delta, t)+12 d>4 e+b-1+8 d .
\end{gathered}
$$

The parameters $a, b$, and $c$ are chosen to be the minimal values in the proper congruence satisfying the analogues of the three conditions mentioned after the statement of 4.1.

We tabulate below the values of $a, b$, and $c$ used in each congruence, the value of $\delta$ used (from 1.1), and the smallest value of $e$ for which there is a $d$ satisfying the two inequalities

\begin{tabular}{cc|cccc|c}
\begin{tabular}{cc|c}
$\boldsymbol{n}$ \\
$\bmod 4$
\end{tabular} & $\bmod 8$ & $a$ & $b$ & $c$ & $\delta$ & smallest $\boldsymbol{c}$ \\
\hline 0 & 0 & 4 & 8 & 9 & 1 & 24 \\
0 & 2 & 6 & 10 & 11 & 1 & 12 \\
0 & 4 & 7 & 12 & 13 & 1 & 16 \\
0 & 6 & 7 & 14 & 13 & 1 & 16 \\
1 & 0 & 6 & 12 & 11 & 3 & 17 \\
1 & 2 & 8 & 14 & 15 & 0 & 17 \\
1 & 4 & 5 & 8 & 9 & 0 & 17 \\
1 & 6 & 9 & 18 & 17 & 3 & 17 \\
2 & 0 & 8 & 16 & 15 & 2 & 18 \\
2 & 2 & 6 & 10 & 13 & -1 & 18 \\
2 & 4 & 7 & 12 & 13 & -1 & 22 \\
2 & 6 & 7 & 14 & 13 & 2 & 18 \\
3 & 0 & 6 & 12 & 11 & 1 & 23 \\
3 & 2 & 8 & 14 & 15 & -1 & 19 \\
3 & 4 & 5 & 8 & 11 & -1 & 23 \\
3 & 6 & 9 & 18 & 17 & 1 & 19
\end{tabular}

The proof of the analogue of 4.1 for $e=20, n \equiv 0$ (8) requires a slight modification. We use $X=P^{\langle t-1\rangle}{ }_{2 e+1}$ but then can lift to $X_{1}$ without using the map $g_{1}$ in the factorization of $\phi$.

Proof of 4.2. $D_{N} P_{k}^{l} \rightarrow^{D_{N} \phi} D_{N} P_{k+8}^{l+8}$ is a map of filtration 4 ; in fact it is stably of the same type as $\phi-\Sigma^{N-L-k} P_{L+k-1}^{L} \rightarrow \Sigma^{N-L-k} P_{L+k-1-8}^{L-8}$ for appropriate $L$. Letting $Z=D_{N} P_{k+8}^{l+8}, D_{N} \phi$ can be factored as

$$
D_{N} P_{k}^{l} \rightarrow Z^{\langle 4\rangle} \rightarrow Z^{\langle 3\rangle} \rightarrow Z^{\langle 2\rangle} \rightarrow Z^{\langle 1\rangle} \rightarrow Z .
$$

If $W_{i}$ denotes the $N$-skeleton $\left(Z^{\langle i\rangle}\right)^{(N)}$, then $D_{N} \phi$ factors as $D_{N} P_{k}^{l} \rightarrow W_{3} \rightarrow W_{2} \rightarrow$ $W_{1} \rightarrow Z$ with each map cohomologically trivial. Applying $D_{N}$ to this sequence yields the sequence stated in 4.3.

To see that (ii) is satisfied, note that

$$
D_{N} Y_{i} \wedge X \wedge b o=\left(\left(\Sigma^{N-L-k} P_{L+k-l-8}^{L-8}\right)^{\langle i\rangle}\right)^{(N)} \wedge P_{2 e+1}^{\langle t\rangle} \wedge b o=U
$$


For $s>0$ and $*-s \leqslant N+2 e$,

$$
\operatorname{Ext}_{\mathscr{Q}}^{s, *}\left(H^{*} U, Z_{2}\right) \approx \operatorname{Ext}_{\mathscr{Q}_{1}}^{s+i+t, *+i+t}\left(H^{*}\left(\Sigma^{N-L-k} P_{L+k-l-8}^{L-8} \wedge P_{2 e+1}\right), Z_{2}\right) .
$$

This was calculated in [4, 3.9 and 3.10]. The map in $\operatorname{Ext}_{\mathscr{Q}}()$ induced by $U \rightarrow$ $\Sigma^{N-L-k} P_{L+k-l-8}^{L-8} \wedge P_{2 e+1} \wedge b o$ is through dimension $N+2 e$ of the type suggested by the charts
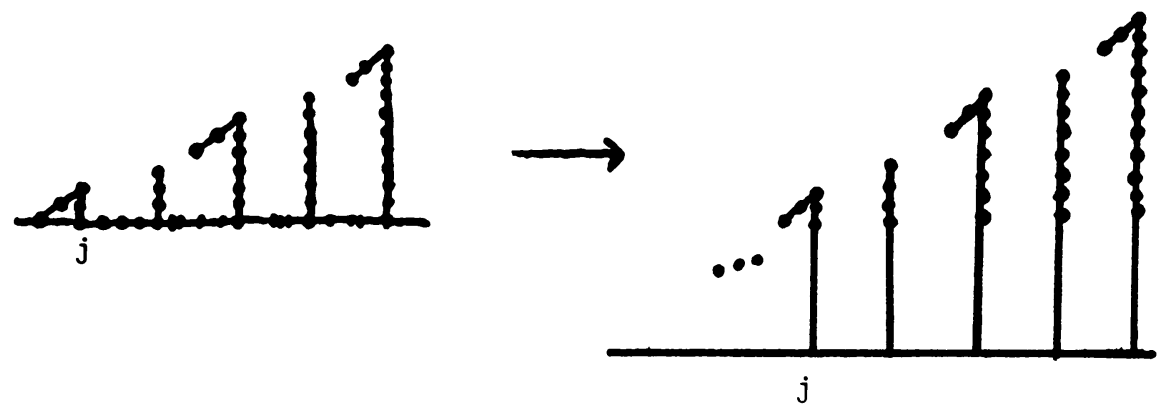

and hence there can be no differentials in $\operatorname{ASS}(U)$ through dimension $N+2 e$.

REMARK 4.4. The proof of 1.1 when $n$ is odd is complicated by the fact that maps $\phi: P_{k+8}^{n+8} \rightarrow P_{k}^{n}$ inducing an isomorphism in $K$-theory do not exist when $n$ is odd. The lifting can be obtained by using the maps $\phi$ for $P^{n+1}$, which is lifted past its $b J$-primary obstruction by varying on the top cell.

Consider for example the case $e=4 e^{\prime}, \quad n=8 l+1$. We must show $\operatorname{gd}\left(2^{4 l+1-4 e^{\prime}} \xi_{8 l+1}\right)=8 e^{\prime}+1$ where

$$
\Sigma^{-1} P^{8 l+2}{\stackrel{24 l+1-4 e^{\prime} \xi}{\rightarrow}}^{8 e^{\prime}+1} \wedge b J
$$

has a nonzero obstruction on the top cell. Let $X=P_{8 e^{\prime}+1}^{\langle t\rangle}$, where $t=4 l+1-4 e^{\prime}$ $-\left[\log _{2}(8 l+2)\right]-2$ (two stages lower than maximal). Then $f^{\prime}: \Sigma^{-1} P^{8 l+2} \rightarrow X$, a lifting of the map which classifies $2^{4 l+1-4 e^{\prime}} \xi$, is cohomologically trivial (because $f^{\prime}$ lifts to $P_{8 e^{\prime}+1}^{\langle t+1\rangle}$. Thus, by the argument in the next-to-last paragraph of the proof of 4.1, $\Sigma^{-1 P^{8 l+2}} \rightarrow^{f^{\prime}} X \rightarrow X \wedge b o$ is trivial so there is a lifting $\Sigma^{-1} P^{8 l+2} \rightarrow^{l} X_{1}$. Since $f^{\prime}$ lifts to $P_{8 e^{\prime}+1}^{\langle t+2\rangle}, l$ can be chosen to be 0 in cohomology. As in the last paragraph of the proof of $4.1, l$ can be chosen so that when followed into $X_{1} \wedge$ bo only its component $c$ into $P_{8 e^{\prime}+1}^{\langle t\rangle} \wedge \Sigma^{3} b s p$ is nonzero. Since the $b J$-primary obstruction for lifting $2^{4 l+1-4 e^{\prime}} \xi_{8 l+1}$ to $8 e^{\prime}+1$ is zero (by $\S 2$ ), $c$ is the composite $\Sigma^{-1} P^{8 l+2}$ $\rightarrow^{p} S^{8 l+1} \rightarrow^{g} P_{8 e^{\prime}+1} \wedge \Sigma^{3} b s p$. Analysis of the upper edge of the Adams charts for

$$
\begin{array}{ccc}
\pi_{*}\left(X_{1}\right) & \rightarrow & \pi_{*}\left(P_{8 e^{\prime}+1} \wedge \Sigma^{3} b s p\right) \\
\downarrow & & \\
\pi_{*}\left(\underline{P}_{8 e^{\prime}+1}^{\langle t\rangle}\right) & \rightarrow & \pi_{*}\left(P_{8 e^{\prime}+1} \wedge b o\right)
\end{array}
$$

shows $g$ is the image of a class $\hat{g} \in \pi_{8 l+1}\left(X_{1}\right)$. Let $\Delta$ denote the composite $\Sigma^{-1} P^{8 l+2} \rightarrow^{p} S^{8 l+1} \rightarrow^{\hat{g}} X_{1}$. Then $l+\Delta$ lifts to $l_{2}$ into $X_{2}$ and $l+\Delta \mid P^{8 l+1}=$ $l \mid P^{8 l+1}$. Now use the method of 4.1 to find $d$ such that $\phi^{d} \circ l_{2}$ is trivial (for dimensional reasons using the bo-resolution) and is in the range where triviality 
into $\underline{P_{8 e^{\prime}+1}^{\langle t\rangle}}$ implies triviality into $\underline{V_{8 e^{\prime}+1}^{\langle t}}$. Thus it suffices to show that

$$
\Sigma^{-1} P^{8 l+1+8 d} \rightarrow \Sigma^{-1} P^{8 l+2+8 d} \stackrel{\phi^{d}}{\rightarrow} \Sigma^{-1} P_{2 m+1}^{8 l+2} \stackrel{l+\Delta}{\rightarrow} X_{1} \stackrel{q}{\rightarrow} Q P_{8 e^{\prime}+1}
$$

agrees with $\Sigma^{-1} P^{8 l+1+8 d} \rightarrow S O \rightarrow Q P_{8 e^{\prime}+1}$ where $\theta$ classifies a bundle of order $2^{e}$. This follows since $\left[\Sigma^{-1} P^{8 l+1+8 d}, Q P_{8 e^{\prime}+1}\right]$ has no nontrivial elements whose filtration is as large as that of $\Delta \circ \phi^{d}$.

\section{REFERENCES}

1. J. F. Adams, A periodicity theorem in homological algebra, Proc. Cambridge Philos. Soc. 62 (1966), 365-377.

2. D. Anderson, E. Brown and F. P. Peterson, The structure of the Spin Cobordism ring, Ann. of Math. 86 (1967), 271-298.

3. E. B. Curtis, Some nonzero homotopy groups of spheres, Bull. Amer. Math. Soc. 75 (1969), 541-544.

4. D. M. Davis, Generalized homology and the generalized vector field problem, Quart. J. Math. Oxford 25 (1974), 169-193.

5. The cohomology of the spectrum bJ, Bol. Soc. Mat. Mexicana 20 (1975), 6-11.

6. D. M. Davis and M. Mahowald, Obstruction theory and ko-theory, Lecture Notes in Math., vol. 658, Springer-Verlag, Berlin and New York, 1978, pp. 134-164.

7. _ A nondesuspension theorem for stunted real projective spaces, Proc. Amer. Math. Soc. 71 (1978), 143-146.

8. S. Feder, S. Gitler and K. Y. Lam, Composition properties of projective homotopy classes, Pacific J. Math. 68 (1977), 47-61.

9. S. Feder and W. Iberkleid, Secondary operations in K-theory and sections of vector bundles, Lecture Notes in Math., vol. 597, Springer-Verlag, Berlin and New York, 1977, pp. 161-175.

10. K. Y. Lam, KO-equivalences and existence of nonsingular bilinear maps, Pacific J. Math. 82 (1979), 145-153.

11. J. M. Long, Two contributions to the homotopy theory of $\boldsymbol{H}$-spaces, Thesis, Princeton University, 1979.

12. M. Mahowald, $A$ new infinite family in ${ }_{2} \pi_{*}^{2}$, Topology 16 (1977), 249-256.

13. _ The metastable homotopy of $S^{n}$, Mem. Amer. Math. Soc., no. 72, Amer. Math. Soc., Providence, R. I., 1967.

14. _ bo-resolutions, Pacific J. Math. (to appear).

15. __ Ring spectra which are Thom complexes, Duke Math. J. 46 (1979), 549-559.

16. M. Mahowald and R. J. Milgram, Operations which detect $\mathrm{Sq}^{4}$ in connective $K$-theory and their applications, Quart. J. Math. Oxford 27 (1976), 415-432.

17. S. Mac Lane, Homology, Academic Press, New York, 1963.

18. R. J. Milgram, The Steenrod algebra and its dual for connective $K$-theory, Notas de Mat. y Simp., Soc. Mat. Mexicana 1 (1975), 127-158.

19. A. Unell, A nonembedding result for an infinite family of homotopy spheres, Thesis, Northwestern University, 1979.

Department of Mathematics, Lehigh University, Bethlehem, Pennsylvania 18015

Centro de InVestigacion del I.P.N., Mexico 14DF, Mexico

Department of Mathematics, NorthWestern University, Evanston, Iluinois 60201 